\title{
A drift-kinetic analytical model for scrape-off layer plasma dynamics at arbitrary collisionality
}

\author{
R. Jorge ${ }^{1,2, \dagger, ~ P . ~}$ Ricci $^{1}$ and N. F. Loureiro ${ }^{3}$ \\ ${ }^{1}$ École Polytechnique Fédérale de Lausanne (EPFL), Swiss Plasma Center (SPC), CH-1015 Lausanne, \\ Switzerland \\ ${ }^{2}$ Instituto de Plasmas e Fusão Nuclear, Instituto Superior Técnico, Universidade de Lisboa, \\ 1049-001 Lisboa, Portugal \\ ${ }^{3}$ Plasma Science and Fusion Center, Massachusetts Institute of Technology, Cambridge, MA 02139, USA
}

(Received 4 September 2017; revised 17 October 2017; accepted 19 October 2017)

A drift-kinetic model to describe the plasma dynamics in the scrape-off layer region of tokamak devices at arbitrary collisionality is derived. Our formulation is based on a gyroaveraged Lagrangian description of the charged particle motion, and the corresponding drift-kinetic Boltzmann equation that includes a full Coulomb collision operator. Using a Hermite-Laguerre velocity space decomposition of the gyroaveraged distribution function, a set of equations to evolve the coefficients of the expansion is presented. By evaluating explicitly the moments of the Coulomb collision operator, distribution functions arbitrarily far from equilibrium can be studied at arbitrary collisionalities. A fluid closure in the high-collisionality limit is presented, and the corresponding fluid equations are compared with previously derived fluid models.

Key words: fusion plasma, plasma confinement, plasma nonlinear phenomena

\section{Introduction}

The success of the magnetic confinement fusion program relies on our ability to predict the dynamics of the plasma in the tokamak scrape-off layer (SOL). In this region, the plasma is turbulent with a fluctuation level of order unity (Ritz et al. 1987; Wootton et al. 1990; Hidalgo et al. 2002; LaBombard et al. 2005; D'Ippolito, Myra \& Zweben 2011). The fluctuations are characterized by frequencies lower than the ion gyrofrequency (Endler et al. 1995; Agostini et al. 2011; Carralero et al. 2014; Garcia, Horacek \& Pitts 2015), and the turbulent eddies, which include coherent radial propagation of filamentary structures (D'Ippolito, Myra \& Krasheninnikov 2002; Carreras 2005; Serianni et al. 2007; D'Ippolito et al. 2011), have a radial extension comparable to the time-averaged SOL pressure gradient length $L_{p}$ (Zweben et al. 2007).

In recent years, there has been a significant development of first-principles simulations of the SOL dynamics with both kinetic (Tskhakaya 2012) and gyrokinetic (Xu et al. 2007; Shi, Hakim \& Hammett 2015; Chang et al. 2017; Shi et al. 2017) codes. However, as kinetic simulations of the SOL and edge regions remain prohibitive as they still are computationally extremely expensive, the less demanding fluid

$\dagger$ Email address for correspondence: rogerio.jorge@epfl.ch 
simulations are the standard of reference (Dudson et al. 2009; Tamain et al. 2009; Easy et al. 2014; Halpern et al. 2016; Madsen et al. 2016). The fluid simulations are usually based on the drift-reduced Braginskii (Braginskii 1965; Zeiler, Drake \& Rogers 1997) or gyrofluid (Ribeiro \& Scott 2008; Held et al. 2016) models to evolve plasma density, fluid velocity and temperature. Fluid models assume that the distribution function is close to a local Maxwellian, and that scale lengths along the magnetic field are longer than the mean free path. However, kinetic simulations show that the plasma distribution function is far from Maxwellian in the SOL region (Lönnroth et al. 2006; Tskhakaya et al. 2008; Battaglia et al. 2014) and that collisionless effects in the SOL might become important (Batishchev et al. 1997). This is expected to be particularly true in ITER and other future devices that will be operated in the high confinement mode (H-mode) regime (Martin, Takizuka \& Group 2008). In such cases, a transport barrier is formed that creates a steep pressure gradient at the plasma edge. If the pressure gradient exceeds a threshold value, edge-localized modes (ELMs) are destabilized (Leonard 2014), expelling large amounts of heat and particles to the wall. Describing structures with such high temperatures (and therefore low collisionality) with respect to the background SOL plasma requires therefore a model that allows for the treatment of arbitrary collision frequencies. Higher moments of the distribution function are needed for a proper SOL description (Hazeltine 1998).

Leveraging the development of previous models (Hammett et al. 1993; Beer \& Hammett 1996; Sugama, Watanabe \& Horton 2001; Ji \& Held 2010; Zocco \& Schekochihin 2011; Hatch et al. 2016; Hirvijoki et al. 2016; Parker 2016; Schekochihin et al. 2016; Mandell, Dorland \& Landreman 2017), we construct here a moment hierarchy to evolve the SOL plasma dynamics. Our model is valid in arbitrary magnetic field geometries and, making use of the full Coulomb collision operator, at arbitrary collision frequencies. The model is derived within a full-F framework, as the amplitude of the background and fluctuating components of the plasma parameters in the SOL have comparable amplitude. We work within the drift approximation (Hinton \& Hazeltine 1976; Cary \& Brizard 2009), which assumes that plasma quantities have typical frequencies that are small compared to the ion gyrofrequency $\Omega_{i}=e B / m_{i}$, and their perpendicular spatial scale is small compared to the ion sound Larmor radius $\rho_{s}=c_{s} / \Omega_{i}$, with $c_{s}^{2}=T_{e} / m_{i}, T_{e}$ the electron temperature, $B$ the magnitude of the magnetic field, $e$ the electron charge, and $m_{i}$ the ion mass. Moreover, we consider a Braginskii ordering (Braginskii 1965), where the species flow velocities are comparable to the ion thermal speed, as opposed to the drift ordering introduced by Mikhailovskii \& Tsypin (1971) and extended and corrected by Catto \& Simakov (2004), where flow velocities are comparable to the diamagnetic drift velocities.

More precisely, denoting $k_{\perp} \sim\left|\nabla_{\perp} \log \phi\right| \sim\left|\nabla_{\perp} \log n\right| \sim\left|\nabla_{\perp} \log T_{e}\right|$ and $\omega \sim\left|\partial_{t} \log \phi\right| \sim\left|\partial_{t} \log n\right| \sim\left|\partial_{t} \log T_{e}\right|$, with $\phi$ the electrostatic potential and $n$ the plasma density, we introduce the ordering parameter $\epsilon$ such that

$$
\epsilon \sim k_{\perp} \rho_{s} \sim \omega / \Omega_{i} \ll 1 .
$$

On the other hand, we let $k_{\perp} L_{p} \sim 1$ since turbulent eddies are observed to have an extension comparable to the scale lengths of the time-averaged quantities. These assumptions are in agreement with experimental measurements of SOL plasmas (LaBombard et al. 2001; Zweben et al. 2004; Myra et al. 2013; Carralero et al. 2014). We also order the electron collision frequency $v_{e i}$ as

$$
\frac{v_{e i}}{\Omega_{i}} \sim \epsilon_{v} \lesssim \epsilon
$$


In addition, the ion collision frequency $v_{i}=v_{i i}$ is ordered as $v_{i i} \lesssim \epsilon^{2} \Omega_{i}$ that, noticing $v_{i} \sim \sqrt{m_{e} / m_{i}}\left(T_{e} / T_{i}\right)^{3 / 2} v_{e}\left(\right.$ with $\left.v_{e}=v_{e i}\right)$, yields

$$
\left(\frac{\epsilon_{v}}{\epsilon^{2}}\right)^{2 / 3}\left(\frac{m_{e}}{m_{i}}\right)^{1 / 3} \lesssim \frac{T_{i}}{T_{e}} \lesssim 1
$$

The ordering in (1.3) can be used to justify applying our model in the cold ion limit, $T_{i} \ll T_{e}$, but allows for $T_{i} \sim T_{e}$. We note that in the SOL the ratio $T_{i} / T_{e}$ is in the range $1 \lesssim T_{i} / T_{e} \lesssim 4$ (Kočan et al. 2011). The ion temperature in this range of values is seen to play a negligible role in determining the SOL turbulent dynamics, usually due to a steeper electron temperature profile compared with the ion one, which is usually below the threshold limit of the ion temperature gradient instability (Mosetto et al. 2015).

The ordering in (1.1)-(1.3) is justified in a wide variety of experimental conditions. For example, for a typical JET discharge (Erents et al. 2000; Liang et al. 2007; Xu et al. 2009) with the SOL parameters $B_{T}=2.5 \mathrm{~T}, T_{e} \sim T_{i} \sim 20 \mathrm{eV}, n_{e} \simeq 10^{19} \mathrm{~m}^{-3}$ and $k_{\perp} \sim 1 \mathrm{~cm}^{-1}$, we obtain $\epsilon_{\nu} \sim 0.016$ and $\epsilon \sim 0.0182$. For a medium-sized tokamak such as TCV (Rossel et al. 2012; Nespoli et al. 2017), estimating $B_{T}=1.5 \mathrm{~T}, T_{e} \sim T_{i} \sim$ $40 \mathrm{eV}, n_{e} \simeq 6 \times 10^{18}$ and $k_{\perp} \sim 1 \mathrm{~cm}^{-1}$, we obtain $\epsilon_{v} \sim 6.2 \times 10^{-3}$ and $\epsilon \sim 0.043$. Finally, for small-sized tokamaks such as ISTTOK (Silva et al. 2011; Jorge et al. 2016), with $B_{T}=0.5 \mathrm{~T}, T_{e} \sim T_{i} \sim 20 \mathrm{eV}, n_{e} \simeq 0.8 \times 10^{18}$ and $k_{\perp} \sim 1 \mathrm{~cm}^{-1}$, we obtain $\epsilon_{\nu} \sim 0.0072$ and $\epsilon \sim 0.091$. Lower values of $\epsilon_{\nu}$, as in the presence of ELMs where temperatures can reach up to $100 \mathrm{eV}$ (Pitts et al. 2003), are also included in the ordering considered here.

Following typical SOL experimental measurements (see, e.g. Zweben et al. (2007), Terry et al. (2009), Grulke et al. (2014)), we order $k_{\|} \sim 1 / L_{B} \sim 1 / R$, with $L_{B}$ the background magnetic field spatial gradient scale and $R$ the tokamak major radius, and take $k_{\|} \rho_{s} \sim \epsilon^{3}$. This yields

$$
\frac{k_{\|}}{k_{\perp}} \sim \epsilon^{2},
$$

a lower ratio than the ones used in most drift-kinetic and gyrokinetic deductions (Hahm 1988; Hazeltine \& Meiss 2003; Abel et al. 2013). The orderings in (1.2) and (1.4) imply that

$$
k_{\|} \lambda_{m f p} \sim \sqrt{\frac{m_{i}}{m_{e}}} \frac{\epsilon^{3}}{\epsilon_{v}}
$$

which includes both the collisional regime $k_{\|} \lambda_{m f p} \lesssim 1$, when $\epsilon_{v} \sim \epsilon$, and the collisionless regime $k_{\|} \lambda_{m f p} \gg 1$, when $\epsilon_{v} \ll \epsilon$. Finally, the plasma parameter $\beta=n T_{e} /\left(B^{2} / 2 \mu_{0}\right)$ is ordered as $\beta \lesssim \epsilon^{3}$, implying that our equations describe plasma dynamics in an electrostatic regime.

Our model describes the evolution of the moments of the drift-kinetic Boltzmann equation at order $\epsilon^{2}$, taking into account the effect of collisions through a full Coulomb collision operator. The kinetic equation is based on a Lagrangian description of the charged particle motion. In the SOL, due to the large fluctuations and the short characteristic gradient width $L_{\phi} \sim L_{p}$, a strong electric field is present. To properly retain the effect of a non-negligible $\boldsymbol{E} \times \boldsymbol{B}$ drift, $\boldsymbol{v}_{E}=-\nabla \phi \times \boldsymbol{B} / B^{2} \quad(\boldsymbol{E}=-\nabla \phi$ in the electrostatic limit employed here), in the equations of motion, we split the perpendicular component of the particle velocity $\boldsymbol{v}_{\perp}$ into $\boldsymbol{v}_{\perp}=\boldsymbol{v}_{E}+\boldsymbol{v}_{\perp}^{\prime}$. In the particle Lagrangian, we keep the resulting term $m v_{E}^{2} / 2$ associated with the $\boldsymbol{E} \times \boldsymbol{B}$ motion of the gyrocentres, as it will be shown to be of the same order of magnitude as the first-order terms in the Lagrangian (see Krommes (2013) for a discussion on the physical interpretation of this term). 
In the kinetic equation, we expand the gyroaveraged distribution function into a Hermite-Laguerre basis, and express the moments of the collision operator in a series of products of the expansion coefficients of the distribution function. For like-species collisions, this expansion is based on the work of Ji \& Held (2009), while we make use of the small mass ratio approximation to obtain electron-ion and ion-electron operators that ensure basic conservation properties. The system is closed by Poisson's equation, involving explicitly the moments of the distribution function, accurate up to order $\epsilon^{2}$ (we also present a derivation of Poisson's equation that rigorously includes collisional $\epsilon_{v}$ effects).

This paper is organized as follows. Section 2 derives the equations of motion of a charged particle in the SOL, and the drift-kinetic Boltzmann equation in a conservative form. In $\S 3$ we expand the gyroaveraged distribution function in a Hermite-Laguerre basis and obtain the guiding-centre moments of the collision operator. In $\S 4$ we take moments of the drift-kinetic Boltzmann equation, and deduce the moment-hierarchy equations. Section 5 presents the guiding-centre Poisson equation, accurate up to order $\epsilon^{2}$. Finally, in $\S 6$, a fluid model based on the truncation of the Hermite-Laguerre expansion in the high-collisionality regime is presented. The conclusions follow. Appendix A presents the transformation between pitch-angle and parallel-perpendicular velocity bases. Appendix B lists explicitly the moments of the parallel acceleration phase-space-conserving term. Appendix C derives Poisson's equation with higher-order collisional effects. Finally, in appendix D, the lower-order guiding-centre moments of the collision operator are given in explicit form.

\section{SOL guiding-centre model}

\subsection{Single particle motion}

To derive a convenient equation of motion in the presence of a strong magnetic field $\boldsymbol{B}$, we start with the Hamiltonian of a charged particle of species $a$ (Jackson 1998),

$$
H_{a}(\boldsymbol{x}, \boldsymbol{p})=\frac{\left[\boldsymbol{p}-q_{a} \boldsymbol{A}\right]^{2}}{2 m_{a}}+q_{a} \phi
$$

and its associated Lagrangian,

$$
L_{a}(\boldsymbol{x}, \boldsymbol{v})=\left[q_{a} \boldsymbol{A}(\boldsymbol{x})+m_{a} \boldsymbol{v}\right] \cdot \dot{\boldsymbol{x}}-\left(\frac{m_{a} v^{2}}{2}+q_{a} \phi(\boldsymbol{x})\right)
$$

where $\boldsymbol{p}=m_{a} \boldsymbol{v}+q_{a} \boldsymbol{A}, \boldsymbol{v}$ is the particle velocity, $\boldsymbol{A}$ is the magnetic vector potential, $\phi$ is the electrostatic potential, $m_{a}$ is the mass of the particle and $q_{a}$ its charge.

We now perform a coordinate transformation from the phase-space coordinates $\boldsymbol{z}=(\boldsymbol{x}, \boldsymbol{v})$ to the guiding-centre coordinates $\boldsymbol{Z}=\left(\boldsymbol{R}, v_{\|}, \mu, \theta\right)$ by writing the particle velocity as (see, e.g. Littlejohn (1983))

$$
\boldsymbol{v}=\boldsymbol{U}+v_{\perp}^{\prime} \boldsymbol{c}
$$

with $\boldsymbol{U}=\boldsymbol{v}_{E}(\boldsymbol{R})+v_{\|} \boldsymbol{b}(\boldsymbol{R}), v_{\|}=\boldsymbol{v} \cdot \boldsymbol{b}, \boldsymbol{b}=\boldsymbol{B} / B$ and $\boldsymbol{v}_{E}=\boldsymbol{E} \times \boldsymbol{B} / B^{2}$. The gyroangle $\theta=$ $\tan ^{-1}\left[(\boldsymbol{v}-\boldsymbol{U}) \cdot \boldsymbol{e}_{2} /(\boldsymbol{v}-\boldsymbol{U}) \cdot \boldsymbol{e}_{1}\right]$ is introduced by defining the right-handed coordinate set 
$\left(\boldsymbol{e}_{1}, \boldsymbol{e}_{2}, \boldsymbol{b}\right)$, such that $\boldsymbol{c}=-\boldsymbol{a} \times \boldsymbol{b}=\boldsymbol{a}^{\prime}(\theta)$, with $\boldsymbol{a}=\cos \theta \boldsymbol{e}_{1}+\sin \theta \boldsymbol{e}_{2}$. The decomposition in (2.3) allows us to isolate the high-frequency gyromotion, contained in the $v_{\perp}^{\prime} \boldsymbol{c}$ term, from the dominant guiding-centre velocity $\boldsymbol{U}$. The adiabatic moment $\mu$ is defined as

$$
\mu=\frac{m_{a} v_{\perp}^{\prime 2}}{2 B},
$$

whereas the guiding-centre position is

$$
\boldsymbol{R}=\boldsymbol{x}-\rho_{a} \boldsymbol{a},
$$

with $\rho_{a}=\sqrt{2 m_{a} \mu /\left(q_{a}^{2} B\right)}$ the Larmor radius. Incidentally, for the case of weakly varying magnetic fields, equation (2.5) describes the circular motion of a particle around its guiding centre $\boldsymbol{R}$ with radius $\rho_{a}$, i.e. $(\boldsymbol{x}-\boldsymbol{R})^{2}=\rho_{a}^{2}$.

As our goal is to develop a model that describes turbulent fluctuations occurring on a spatial scale longer than the sound Larmor radius $\rho_{s}$, and a time scale larger than the gyromotion one, we keep terms in the Lagrangian up to $O(\epsilon)$ and order $T_{i} \lesssim T_{e}$, which implies

$$
k_{\perp} \rho_{i} \lesssim \epsilon .
$$

We therefore expand the electromagnetic fields around $\boldsymbol{R}$, to first order in $\epsilon$, i.e.

$$
\phi(\boldsymbol{x}) \simeq \phi(\boldsymbol{R})+\rho_{a} \boldsymbol{a} \cdot \nabla_{\boldsymbol{R}} \phi(\boldsymbol{R}),
$$

and similarly for $\boldsymbol{A}$. In the following, if not specified, the electromagnetic fields and potentials are evaluated at the guiding-centre position $R$, and we denote $\nabla=\nabla_{R}$. In addition, to take advantage of the difference between the turbulent and gyromotion time scales, we use the gyroaveraged Lagrangian $\left\langle L_{a}\right\rangle$ to evaluate the plasma particle motion, where the gyroaveraging operator $\langle\chi\rangle$ acting on a quantity $\chi(\theta)$ is defined as

$$
\langle\chi\rangle=\frac{1}{2 \pi} \int_{0}^{2 \pi} \chi(\theta) \mathrm{d} \theta,
$$

which is performed at fixed position $\boldsymbol{x}$, as opposed to the gyrokinetic equation that can be obtained by gyroaveraging with $\boldsymbol{R}$ fixed (Hazeltine \& Meiss 2003).

To evaluate $\left\langle L_{a}\right\rangle$ we note that, with the expansion for $\phi$ and $\boldsymbol{A}$, the Lagrangian in (2.2) can be expressed as $L_{a}=L_{0 a}+L_{1 a}+\tilde{L}_{a}$ where $L_{0 a}$ is gyroangle independent,

$$
L_{0 a}=\left(q_{a} \boldsymbol{A}+m_{a} \boldsymbol{U}\right) \cdot \dot{\boldsymbol{R}}-\left(\frac{m_{a} v_{\|}^{2}}{2}+\frac{m_{a} v_{E}^{2}}{2}+\mu B+q_{a} \phi\right),
$$

$L_{1 a}$ is proportional to $\rho_{a}^{2}$ (and hence to $\mu$ ),

$$
L_{1 a}=\rho_{a}^{2} q_{a} \dot{\theta}(\boldsymbol{a} \cdot \nabla)(\boldsymbol{A} \cdot \boldsymbol{c})+m_{a} \rho_{a}^{2} \Omega \dot{\theta}+\rho_{a} \dot{\rho}_{a}\left[q_{a}(\boldsymbol{a} \cdot \nabla)(\boldsymbol{A} \cdot \boldsymbol{a})\right],
$$

and the $\tilde{L}_{a}$ contribution contains the terms linearly proportional to $\cos \theta$ or $\sin \theta$ (Cary \& Brizard 2009) which are not present in $\left\langle L_{a}\right\rangle$ as $\left\langle\tilde{L}_{a}\right\rangle=0$.

We note that $\left\langle L_{1 a}\right\rangle$ can be simplified since $\langle(\boldsymbol{a} \cdot \boldsymbol{\nabla}) \boldsymbol{A} \cdot \boldsymbol{c}\rangle=-\boldsymbol{b} \cdot(\boldsymbol{\nabla} \times \boldsymbol{A}) / 2$, and $\langle(\boldsymbol{a} \cdot \nabla) \boldsymbol{A} \cdot \boldsymbol{a}\rangle=\nabla_{\perp} \cdot \boldsymbol{A} / 2$. Subtracting the total derivative $-q_{a} \mathrm{~d} / \mathrm{d} t\left(\rho_{a}^{2} \boldsymbol{\nabla}_{\perp} \boldsymbol{A}\right) / 4$ from $\left\langle L_{a}\right\rangle$, which does not alter the resulting equations of motion, we redefine the gyroaveraged Lagrangian as 


$$
\begin{aligned}
\left\langle L_{a}\right\rangle= & \left(q_{a} \boldsymbol{A}+m_{a} \boldsymbol{U}\right) \cdot \dot{\boldsymbol{R}}-\left(\frac{m_{a} v_{\|}^{2}}{2}+\frac{m_{a} v_{E}^{2}}{2}+q_{a} \phi\right) \\
& -\mu B\left(1-\frac{\dot{\theta}}{\Omega_{a}}\right)-\frac{\rho_{a}^{2}}{4} \frac{\mathrm{d}}{\mathrm{d} t}\left[\nabla_{\perp} \cdot\left(q_{a} \boldsymbol{A}\right)\right] .
\end{aligned}
$$

We now order the terms appearing in $\left\langle L_{a}\right\rangle$. As imposed by the Bohm sheath conditions (Stangeby 2000), both electrons and ions stream along the field lines with parallel velocities comparable to the sound speed $c_{s}=\sqrt{T_{e} / m_{i}}$ in the SOL. The Bohm boundary conditions at the sheath also set the electrostatic potential $e \phi \sim \Lambda T_{e}$ across the SOL, where $\Lambda=\log \sqrt{m_{i} /\left(m_{e} 2 \pi\right)} \simeq 3$. Therefore, we keep the $m_{a} v_{E}^{2} / 2$ term in the Lagrangian in (2.11), as to take into account the presence of the numerically large factor $\Lambda^{2}$ in $v_{E}^{2} \sim \epsilon^{2} \Lambda^{2} c_{s}^{2}$.

By neglecting the higher-order terms in (2.11), i.e. $-\left(\rho_{a}^{2} / 4\right) \mathrm{d}\left[\nabla_{\perp} \cdot\left(q_{a} \boldsymbol{A}\right)\right] / \mathrm{d} t$, the expression for the gyroaveraged Lagrangian describing SOL single particle dynamics, up to $O(\epsilon)$, can be written as

$$
\left\langle L_{a}\right\rangle=q_{a} \boldsymbol{A}^{*} \cdot \dot{\boldsymbol{R}}-q_{a} \boldsymbol{\phi}^{*}-\frac{m_{a} v_{\|}^{2}}{2}+\mu \frac{m_{a} \dot{\theta}}{q_{a}},
$$

where $q_{a} \boldsymbol{\phi}^{*}=q_{a} \phi+m_{a} v_{E}^{2} / 2+\mu B$, and $q_{a} \boldsymbol{A}^{*}=q_{a} \boldsymbol{A}+m_{a} v_{\|} \boldsymbol{b}+m_{a} \boldsymbol{v}_{E}$. The EulerLagrange equations applied to the Lagrangian in (2.12) for the coordinates $\theta, v_{\|}$, and $\mu$, yield, respectively, $\dot{\mu}=0, v_{\|}=\boldsymbol{b} \cdot \dot{\boldsymbol{R}}$, and $\dot{\theta}=\Omega_{a}$. For the $\boldsymbol{R}$ coordinate, we obtain

$$
m_{a} \dot{v}_{\|} \boldsymbol{b}=q_{a}\left(\boldsymbol{E}^{*}+\dot{\boldsymbol{R}} \times \boldsymbol{B}^{*}\right),
$$

where the relation $\left(\nabla \boldsymbol{A}-(\nabla \boldsymbol{A})^{\mathrm{T}}\right) \cdot \dot{\boldsymbol{R}}=\dot{\boldsymbol{R}} \times(\nabla \times \boldsymbol{A})$ has been used, and we defined $\boldsymbol{E}^{*}=-\nabla \phi^{*}-\partial_{t} \boldsymbol{A}^{*}$, and $\boldsymbol{B}^{*}=\boldsymbol{\nabla} \times \boldsymbol{A}^{*}$, with the parallel component of $\boldsymbol{B}^{*}$ given by

$$
B_{\|}^{*}=\boldsymbol{B}^{*} \cdot \boldsymbol{b}=B+\frac{m_{a}}{q_{a}} \boldsymbol{b} \cdot \nabla \times\left(v_{\|} \boldsymbol{b}+\boldsymbol{v}_{E}\right) .
$$

By projecting (2.13) along $\boldsymbol{B}^{*}$, we derive $m \dot{v}_{\|} B_{\|}^{*}=e \boldsymbol{E}^{*} \cdot \boldsymbol{B}^{*}$, while crossing with $\boldsymbol{b}$ yields the guiding-centre velocity $\dot{\boldsymbol{R}} B_{\|}^{*}=v_{\|} \boldsymbol{B}^{*}+\boldsymbol{E}^{*} \times \boldsymbol{B} / B$. Using the expressions for the fields $\boldsymbol{E}^{*}$ and $\boldsymbol{B}^{*}$, we obtain

$$
\dot{\boldsymbol{R}}=\boldsymbol{U}+\frac{\boldsymbol{B}}{\Omega_{a} B_{\|}^{*}} \times\left(\frac{\mathrm{d} \boldsymbol{U}}{\mathrm{d} t}+\frac{\mu \nabla B}{m_{a}}\right),
$$

and

$$
m_{a} \dot{v}_{\|}=q_{a} E_{\|}-\mu \nabla_{\|} B+m_{a} \boldsymbol{v}_{E} \cdot \frac{\mathrm{d} \boldsymbol{b}}{\mathrm{d} t}-m_{a} \mathcal{A}
$$

In (2.15), (2.16), in addition to the time derivatives of the phase-space coordinates $\dot{\boldsymbol{R}}, \dot{v}_{\|}$, that only have an explicit time dependence, we define the total derivative $\mathrm{d} / \mathrm{d} t$ of a field $\phi(\boldsymbol{R}, t)$ that has an explicit time and $\boldsymbol{R}$ dependence as $\mathrm{d} \phi / \mathrm{d} t \equiv \partial_{t} \phi+\boldsymbol{U} \cdot \nabla \phi$. The $\mathcal{A}$ term represents the higher-order nonlinear terms in $\dot{v}_{\|}$that ensure phase-space conservation properties (Cary \& Brizard 2009), and it is given by

$$
\mathcal{A}=\frac{B}{B_{\|}^{*}}\left(\left.\frac{\mathrm{d} \boldsymbol{U}}{\mathrm{d} t}\right|_{\perp}+\mu \nabla_{\perp} B\right) \cdot \frac{\nabla \times \boldsymbol{U}}{\Omega_{a}} .
$$


The guiding-centre equations of motion (2.15) and (2.16) satisfy the energy, $E_{g c}=$ $q_{a} \phi^{*}+m_{a} v_{\|}^{2} / 2$ (Cary \& Brizard 2009), and momentum, $\boldsymbol{P}_{g c}=e \boldsymbol{A}^{*}$ (Cary \& Brizard 2009), conservation laws, i.e.

$$
\frac{\mathrm{d} E_{g c}}{\mathrm{~d} t}=q_{a} \frac{\partial \phi^{*}}{\partial t}-q_{a} \frac{\partial \boldsymbol{A}^{*}}{\partial t} \cdot \dot{\boldsymbol{R}}
$$

and

$$
\frac{\partial \boldsymbol{P}_{g c}}{\partial t}=-q_{a} \nabla \phi^{*}+q_{a} \nabla \boldsymbol{A}^{*} \cdot \dot{\boldsymbol{R}} .
$$

In addition, we note that using (2.15), (2.16) and Maxwell's equations, a conservation equation for $B_{\|}^{*}$ can be derived:

$$
\frac{\partial B_{\|}^{*}}{\partial t}+\nabla \cdot\left(\dot{\boldsymbol{R}} B_{\|}^{*}\right)+\frac{\partial}{\partial v_{\|}}\left(\dot{v}_{\|} B_{\|}^{*}\right)=0 .
$$

\subsection{The guiding-centre Boltzmann equation}

The Boltzmann equation for the evolution of the distribution function $f_{a}(\boldsymbol{x}, \boldsymbol{v})$ of the particles in $(\boldsymbol{x}, \boldsymbol{v})$ coordinates is

$$
\frac{\partial f_{a}}{\partial t}+\dot{\boldsymbol{x}} \cdot \nabla_{x} f_{a}+\dot{\boldsymbol{v}} \cdot \nabla_{v} f_{a}=C\left(f_{a}\right)
$$

where $C\left(f_{a}\right)=\sum_{b} C\left(f_{a}, f_{b}\right)=\sum_{b} C_{a b}$ is the collision operator. Because $f_{a}$ can significantly deviate from a Maxwellian distribution function in the SOL (Battaglia et al. 2014), we consider the bilinear Coulomb operator $C_{a b}$ (Balescu 1988), to model collisions between particles of species $a$ and $b$

$$
C_{a b}=L_{a b} \frac{\partial}{\partial v_{i}}\left[\frac{\partial^{2} G_{b}}{\partial v_{i} \partial v_{j}} \frac{\partial f_{a}}{\partial v_{j}}-\frac{m_{a}}{m_{b}} \frac{\partial H_{b}}{\partial v_{i}} f_{a}\right],
$$

with

$$
H_{b}=2 \int \frac{f_{b}\left(\boldsymbol{v}^{\prime}\right)}{\left|\boldsymbol{v}-\boldsymbol{v}^{\prime}\right|} \mathrm{d} \boldsymbol{v}^{\prime}
$$

and

$$
G_{b}=\int f_{b}\left(\boldsymbol{v}^{\prime}\right)\left|\boldsymbol{v}-\boldsymbol{v}^{\prime}\right| \mathrm{d} \boldsymbol{v}^{\prime}
$$

the Rosenbluth potentials satisfying $\nabla_{v}^{2} G_{b}=H_{b}$. In (2.22) we introduced $L_{a b}=$ $q_{a}^{2} q_{b}^{2} \lambda /\left(4 \pi \epsilon_{0}^{2} m_{a}^{2}\right)=v_{a b} v_{\text {tha }}^{3} / n_{b}$, where $\lambda$ is the Coulomb logarithm, $v_{a b}$ the collision frequency between species $a$ and $b$ and $v_{\text {tha }}^{2}=2 T_{a} / m_{a}$.

Taking advantage of the small electron to ion mass ratio, the collision operator between unlike species can be simplified (see, e.g. Balescu (1988), Helander \& Sigmar (2005)). The electron-ion collisions are modelled by using the operator $C_{e i}\left(f_{e}\right)=C_{e i}^{0}+$ $C_{e i}^{1}$, where $C_{e i}^{0}$ is the Lorentz pitch-angle scattering operator

$$
C_{e i}^{0}=\frac{n_{i} L_{e i}}{8 \pi} \frac{\partial}{\partial \boldsymbol{c}_{e}} \cdot\left[\frac{1}{c_{e}} \frac{\partial f_{e}}{\partial \boldsymbol{c}_{e}}-\frac{\boldsymbol{c}_{e}}{c_{e}^{3}}\left(\boldsymbol{c}_{e} \cdot \frac{\partial f_{e}}{\partial \boldsymbol{c}_{e}}\right)\right],
$$


and $C_{e i}^{1}$ the momentum-conserving term

$$
C_{e i}^{1}=\frac{n_{i} L_{e i}}{4 \pi c_{e}^{3}} f_{M e} \boldsymbol{u}_{i} \cdot c_{e}
$$

with $\boldsymbol{c}_{e}=\left(\boldsymbol{v}-\boldsymbol{u}_{e}\right) / v_{\text {the }}$ the normalized difference between the electron particle $\boldsymbol{v}$ and fluid $\boldsymbol{u}_{e}$ velocities, and $\boldsymbol{u}_{i}$ the ion fluid velocity.

Ion-electron collisions are modelled with the operator

$$
C_{i e}=\frac{\boldsymbol{R}_{e i}}{m_{i} n_{i} v_{t h i}} \cdot \frac{\partial f_{i}}{\partial \boldsymbol{c}_{i}}+v_{e i} \frac{n_{e}}{n_{i}} \frac{m_{e}}{m_{i}} \frac{\partial}{\partial \boldsymbol{c}_{i}} \cdot\left(\boldsymbol{c}_{i} f_{i}+\frac{T_{e}}{T_{i}} \frac{\partial f_{i}}{\partial \boldsymbol{c}_{i}}\right),
$$

where $\boldsymbol{R}_{e i}=\int m_{e} \boldsymbol{v} C_{e i} \mathrm{~d} \boldsymbol{v}$ is the electron-ion friction force.

We take advantage of (1.2) to order the electron collision frequency $v_{e}$ and the ion collision frequency $v_{i}$ as

$$
\frac{v_{i}}{\Omega_{i}} \sim \sqrt{\frac{m_{e}}{m_{i}}}\left(\frac{T_{e}}{T_{i}}\right)^{3 / 2} \epsilon_{\nu} \lesssim \epsilon^{2}
$$

where we used the relation $v_{i} \sim \sqrt{m_{e} / m_{i}}\left(T_{e} / T_{i}\right)^{3 / 2} v_{e}$. The orderings in (2.7) and (2.28) yield the lower bound in (1.3) for the ion to electron temperature ratio. We now express the particle distribution function $f_{a}$ in terms of the guiding-centre coordinates by defining $F_{a}$, a function of guiding-centre coordinates, as

$$
F_{a}\left(\boldsymbol{R}, v_{\|}, \mu, \theta\right)=f_{a}\left(\boldsymbol{x}\left(\boldsymbol{R}, v_{\|}, \mu, \theta\right), \boldsymbol{v}\left(\boldsymbol{R}, v_{\|}, \mu, \theta\right)\right)
$$

Using the chain rule to rewrite (2.21) in guiding-centre coordinates, we obtain

$$
\frac{\partial F_{a}}{\partial t}+\dot{\boldsymbol{R}} \cdot \nabla F_{a}+\dot{v}_{\|} \frac{\partial F_{a}}{\partial v_{\|}}+\dot{\mu} \frac{\partial F_{a}}{\partial \mu}+\dot{\theta} \frac{\partial F_{a}}{\partial \theta}=C\left(F_{a}\right),
$$

where $\dot{\boldsymbol{R}}$ and $\dot{v}_{\|}$are given by (2.15) and (2.16) respectively, $\dot{\theta}=\Omega_{a}$, and $\dot{\mu}=0$. Equation (2.30) can be simplified by applying the gyroaveraging operator. This results in the drift-kinetic equation

$$
\frac{\partial\left\langle F_{a}\right\rangle}{\partial t}+\dot{R} \cdot \nabla\left\langle F_{a}\right\rangle+\dot{v}_{\|} \frac{\partial\left\langle F_{a}\right\rangle}{\partial v_{\|}}=\left\langle C\left(F_{a}\right)\right\rangle .
$$

We now write (2.31) in a form useful to take gyrofluid moments of the form $\int\left\langle F_{a}\right\rangle B \mathrm{~d} v_{\|} \mathrm{d} \mu \mathrm{d} \theta$ (see $\S 4$ ). Using the conservation law in (2.20) for $B_{\|}^{*}$, we can write the guiding-centre Boltzmann equation in conservative form as

$$
\frac{\partial\left(B_{\|}^{*}\left\langle F_{a}\right\rangle\right)}{\partial t}+\nabla \cdot\left(\dot{\boldsymbol{R}} B_{\|}^{*}\left\langle F_{a}\right\rangle\right)+\frac{\partial\left(\dot{v}_{\| a} B_{\|}^{*}\left\langle F_{a}\right\rangle\right)}{\partial v_{\|}}=B_{\|}^{*}\left\langle C\left(F_{a}\right)\right\rangle .
$$

Moreover, in order to relate the gyrofluid moments $\int\left\langle F_{a}\right\rangle B \mathrm{~d} v_{\|} \mathrm{d} \mu \mathrm{d} \theta$ with the usual fluid moments $\int f_{a} \mathrm{~d}^{3} v$, we estimate the order of magnitude of the gyrophase dependent part of the distribution function $\tilde{F}_{a}=F_{a}-\left\langle F_{a}\right\rangle$ where $\left\langle F_{a}\right\rangle$ obeys (2.31). 
The equation for the evolution of $\tilde{F}_{a}$ is obtained by subtracting (2.31) from the Boltzmann equation, (2.30), that is

$$
\frac{\partial \tilde{F}_{a}}{\partial t}+\dot{\boldsymbol{R}} \cdot \nabla \tilde{F}_{a}+\dot{v}_{\|} \frac{\partial \tilde{F}_{a}}{\partial v_{\|}}+\Omega_{a} \frac{\partial \tilde{F}_{a}}{\partial \theta}=C\left(F_{a}\right)-\left\langle C\left(F_{a}\right)\right\rangle .
$$

Using the orderings in (1.2) and (2.28), as well as $\partial_{t} \sim \dot{\boldsymbol{R}} \cdot \nabla \sim \dot{v}_{\|} \partial_{v_{\|}} \sim \epsilon \Omega_{i}$ and $\Omega_{a} \partial_{\theta} \sim \Omega_{a}$, the comparison of the leading-order term on the left-hand side of (2.33) with the right-hand side of the same equation imply the following ordering for $\tilde{F}_{e}$

$$
\frac{\tilde{F}_{e}}{\left\langle F_{e}\right\rangle} \sim \frac{m_{e}}{m_{i}} \epsilon_{v} \lesssim \epsilon^{2}
$$

and $\tilde{F}_{i}$

$$
\frac{\tilde{F}_{i}}{\left\langle F_{i}\right\rangle} \sim \sqrt{\frac{m_{e}}{m_{i}}}\left(\frac{T_{e}}{T_{i}}\right)^{3 / 2} \epsilon_{v} \lesssim \epsilon^{2}
$$

To evaluate the leading-order term of $\tilde{F}_{a}$, we expand the collision operator $C\left(F_{a}\right)=$ $C_{0}\left(\left\langle F_{a}\right\rangle\right)+\epsilon C_{1}\left(F_{a}\right)+\cdots$, such that

$$
\tilde{F}_{a} \simeq \frac{1}{\Omega_{a}} \int_{0}^{\theta}\left[C_{0}\left(\left\langle F_{a}\right\rangle\right)-\left\langle C_{0}\left(\left\langle F_{a}\right\rangle\right)\right\rangle\right] \mathrm{d} \theta^{\prime}+O\left(\epsilon^{3}\left\langle F_{a}\right\rangle\right) .
$$

The relation in (2.36) can be further simplified by expanding the $\theta$ dependence of $F_{a}$ in Fourier harmonics,

$$
F_{a}=\sum_{m} \mathrm{e}^{\mathrm{i} m \theta} F_{m a}
$$

so that for $m=0$ we have $\left\langle F_{a}\right\rangle=F_{0 a}$, and similarly for $C_{0}\left(\left\langle F_{a}\right\rangle\right)$

$$
C_{0}\left(\left\langle F_{a}\right\rangle\right)=\sum_{m^{\prime}} \mathrm{e}^{\mathrm{i} m^{\prime} \theta} C_{m^{\prime} a} .
$$

We can then write (2.36) as

$$
\tilde{F}_{m a}=\frac{C_{m a}}{\mathrm{i} m \Omega_{a}}
$$

for $m \neq 0$.

\section{Moment expansion}

We now derive a polynomial expansion for the distribution function $\left\langle F_{a}\right\rangle$ that simplifies the solution of (2.32), with the collision operators in (2.22)-(2.27). This section is organized as follows. In $\S 3.1$ the Hermite-Laguerre basis is introduced, relating the corresponding expansion coefficients for $\left\langle F_{a}\right\rangle$ with its usual gyrofluid moments. In $\$ 3.2$, we briefly review the fluid moment expansion of the Coulomb collision operator presented in Ji \& Held $(2006,2008)$. In $\S 3.3$, leveraging the work in Ji \& Held (2006, 2008), we expand $C_{a b}$ in terms of the product of the gyrofluid moments, for both like- and unlike-species collisions. This ultimately gives us the possibility of solving (2.32) in terms of gyrofluid moments. 


\subsection{Guiding-centre moment expansion of $\left\langle F_{a}\right\rangle$}

To take advantage of the anisotropy introduced by a strong magnetic field, and efficiently treat the left-hand side of (2.32) where the parallel and perpendicular directions appear decoupled, we express $\left\langle F_{a}\right\rangle$ by using a Hermite polynomial basis expansion for the parallel velocity coordinate (Grad 1949; Armstrong 1967; Grant \& Feix 1967; Ng, Bhattacharjee \& Skiff 1999; Zocco \& Schekochihin 2011; Loureiro, Schekochihin \& Zocco 2013; Parker \& Dellar 2015; Schekochihin et al. 2016; Tassi 2016) and a Laguerre polynomial basis for the perpendicular velocity coordinate (Omotani et al. 2015; Zocco et al. 2015; Mandell et al. 2017). More precisely, we use the following expansion

$$
\left\langle F_{a}\right\rangle=\sum_{p, j=0}^{\infty} \frac{N_{a}^{p j}}{\sqrt{2^{p} p !}} F_{M a} H_{p}\left(s_{\| a}\right) L_{j}\left(s_{\perp a}^{2}\right),
$$

where the physicists' Hermite polynomials $H_{p}$ of order $p$ are defined by Abramowicz et al. (1988)

$$
H_{p}(x)=(-1)^{p} \mathrm{e}^{x^{2}} \frac{\mathrm{d}^{p}}{\mathrm{~d} x^{p}} \mathrm{e}^{-x^{2}}
$$

and normalized via

$$
\int_{-\infty}^{\infty} \mathrm{d} x H_{p}(x) H_{p^{\prime}}(x) \mathrm{e}^{-x^{2}}=2^{p} p ! \sqrt{\pi} \delta_{p p^{\prime}},
$$

and the Laguerre polynomials $L_{j}$ of order $j$ are defined by Abramowicz et al. (1988)

$$
L_{j}(x)=\frac{\mathrm{e}^{x}}{j !} \frac{\mathrm{d}^{j}}{\mathrm{~d} x^{j}}\left(\mathrm{e}^{-x} x^{j}\right),
$$

which are orthonormal with respect to the weight $\mathrm{e}^{-x}$

$$
\int_{0}^{\infty} \mathrm{d} x L_{j}(x) L_{j^{\prime}}(x) \mathrm{e}^{-x}=\delta_{j j^{\prime}} .
$$

Because of the orthogonality of the Hermite-Laguerre basis, the coefficients $N_{a}^{p j}$ of the expansion in (3.1) are

$$
N_{a}^{p j}=\frac{1}{N_{a}} \int \frac{H_{p}\left(s_{\| a}\right) L_{j}\left(s_{\perp a}^{2}\right)\left\langle F_{a}\right\rangle}{\sqrt{2^{p} p !}} \frac{B}{m_{a}} \mathrm{~d} \mu \mathrm{d} v_{\|} \mathrm{d} \theta,
$$

and correspond to the guiding-centre moments of $\left\langle F_{a}\right\rangle$.

In (3.1), the shifted bi-Maxwellian is introduced

$$
F_{M a}=N_{a} \frac{\mathrm{e}^{-s_{\| a}^{2}-s_{\perp a}^{2}}}{\pi^{3 / 2} v_{t h \| a} v_{t h \perp a}^{2}},
$$

where $s_{\| a}$ and $s_{\perp a}$ are the normalized parallel and perpendicular shifted velocities respectively, defined by

$$
s_{\| a}=\frac{v_{\|}-u_{\| a}}{v_{t h \| a}}, \quad v_{t h \| a}^{2}=\frac{2 T_{\| a}}{m_{a}},
$$


and

$$
s_{\perp a}^{2}=\frac{v_{\perp}^{\prime 2}}{v_{t h \perp a}^{2}}=\frac{\mu B}{T_{\perp a}}, \quad v_{t h \perp a}^{2}=\frac{2 T_{\perp a}}{m_{a}},
$$

which provide an efficient representation of the distribution function in both the weak $\left(u_{\| a} \ll v_{\text {tha }}\right)$ and strong flow $\left(u_{\| a} \sim v_{\text {tha }}\right)$ regimes.

The guiding-centre density $N_{a}$, appearing in (3.7), the guiding-centre fluid velocity $u_{\| a}$, in (3.8) and the guiding-centre parallel $T_{\| a}=P_{\| a} / N_{a}$ and perpendicular $T_{\perp a}=$ $P_{\perp a} / N_{a}$ temperatures in (3.8), (3.9) are defined as $N_{a}=\|1\|_{a}, N_{a} u_{\| a}=\left\|v_{\|}\right\|_{a}, P_{\| a}=$ $m_{a}\left\|\left(v_{\|}-u_{\| a}\right)^{2}\right\|_{a}$ and $P_{\perp a}=\|\mu B\|_{a}$, where

$$
\|\chi\|_{a} \equiv \int \chi\left\langle F_{a}\right\rangle \frac{B}{m_{a}} \mathrm{~d} \mu \mathrm{d} v_{\|} \mathrm{d} \theta .
$$

The definitions of $N_{a}, u_{\| a}, P_{\| a}$ and $P_{\perp a}$ imply that $N_{a}^{00}=1, N_{a}^{10}=0, N_{a}^{20}=0, N_{a}^{01}=$ 0 respectively. Later, we will consider the parallel and perpendicular heat flux, defined as

$$
Q_{\| a}=m_{a}\left\|\left(v_{\|}-u_{\| a}\right)^{3}\right\|_{a}, \quad Q_{\perp a}=\left\|\left(v_{\|}-u_{\| a}\right) \mu B\right\|_{a},
$$

which are related to the coefficients $N_{a}^{30}, N_{a}^{11}$ by

$$
N_{a}^{30}=\frac{Q_{\| a}}{\sqrt{3} P_{\| a} v_{\text {tha }}}, \quad N_{a}^{11}=-\frac{\sqrt{2} Q_{\perp a}}{P_{\perp a} v_{\text {tha }}} .
$$

\subsection{Fluid moment expansion of the collision operator}

A polynomial expansion of the collision operators in (2.22) was carried out in Ji \& Held (2006), and later extended to effectively take into account finite fluid velocity and unlike-species collisions in Ji \& Held (2008). This allowed expressing $C_{a b}$ as products of fluid moments of $f_{a}$ and $f_{b}$. We summarize here the main steps of $\mathrm{Ji} \&$ Held (2006, 2008).

Similarly to (3.1), the particle distribution function $f_{a}$ is expanded as

$$
f_{a}=f_{a M} \sum_{l, k=0}^{\infty} \frac{L_{k}^{l+1 / 2}\left(c_{a}^{2}\right) \boldsymbol{P}^{l}\left(\boldsymbol{c}_{a}\right) \cdot \boldsymbol{M}_{a}^{l k}}{\sqrt{\sigma_{k}^{l}}},
$$

where $f_{a M}=n_{a} \exp \left(-c_{a}^{2}\right) /\left(\pi^{3 / 2} v_{\text {tha }}^{3}\right)$ is a shifted Maxwell-Boltzmann distribution function, and $\boldsymbol{c}_{a}$ the shifted velocity defined as $\boldsymbol{c}_{a}=\left(\boldsymbol{v}-\boldsymbol{U}_{a}\right) / v_{\text {tha }}$, with $\boldsymbol{U}_{a}$ the fluid velocity. The fluid variables $n_{a}, \boldsymbol{U}_{a}$, and $T_{a}$ are defined as the usual moments of the particle distribution function $f_{a}$, i.e. $n_{a}=\int f_{a} \mathrm{~d}^{3} \boldsymbol{v}, n_{a} \boldsymbol{U}_{a}=\int f_{a} \boldsymbol{v} \mathrm{d}^{3} v, n_{a} T_{a}=$ $\int m f_{a}\left(\boldsymbol{v}-\boldsymbol{u}_{a}\right)^{2} \mathrm{~d}^{3} v / 3$.

The tensors $\boldsymbol{P}_{a}^{l k}\left(\boldsymbol{c}_{a}\right)=\boldsymbol{P}^{l}\left(\boldsymbol{c}_{a}\right) L_{k}^{l+1 / 2}\left(c_{a}^{2}\right)$ constitute an orthogonal basis, where $\boldsymbol{P}^{l}\left(\boldsymbol{c}_{a}\right)$ is the symmetric and traceless tensor

$$
\boldsymbol{P}^{l}\left(\boldsymbol{c}_{a}\right)=\sum_{i=0}^{\lfloor l / 2\rfloor} d_{i}^{l} S_{i}^{l} c_{a}^{2 i}\left\{\boldsymbol{I}^{i} \boldsymbol{c}_{a}^{l-2 i}\right\}
$$


with $\boldsymbol{I}$ denoting the identity matrix, $\left\{\boldsymbol{A}^{i}\right\}$ denoting the symmetrization of the tensor $\boldsymbol{A}^{i}$, $\lfloor l / 2\rfloor$ denoting the largest integer less than or equal to $l / 2$, and the coefficients $d_{i}^{l}$ and $S_{i}^{l}$ defined by

$$
d_{i}^{l}=\frac{(-2)^{i}(2 l-2 i) ! l !}{(2 l) !(l-i) !}
$$

and

$$
S_{i}^{l}=\frac{l !}{(l-2 i) ! 2^{i} i !} .
$$

The tensor $\boldsymbol{P}^{l}\left(\boldsymbol{c}_{a}\right)$ is normalized via

$$
\int \mathrm{d} \boldsymbol{v} \boldsymbol{P}^{n}(\boldsymbol{v}) \boldsymbol{P}^{l}(\boldsymbol{v}) \cdot \boldsymbol{M}^{l} g(v)=\boldsymbol{M}^{n} \delta_{n, l} \sigma_{n} \int \mathrm{d} \boldsymbol{v} v^{2 n} g(v),
$$

with $\sigma_{l}=l ! /\left[2^{l}(l+1 / 2) !\right]$. We note that the tensor $\boldsymbol{A}^{i}$ is formed by $i$ multiplications of the $\boldsymbol{A}$ elements (e.g. if $\boldsymbol{A}$ is a rank-2 tensor, $\boldsymbol{A}^{3} \equiv \boldsymbol{A A \boldsymbol { A }}$, which in index notation can be written as $\left.\left(A^{3}\right)_{i j l k m n}=A_{i j} A_{l k} A_{m n}\right)$.

In the expansion in (3.13), $L_{k}^{l+1 / 2}(x)$ are the associated Laguerre polynomials

$$
L_{k}^{l+1 / 2}(x)=\sum_{m=0}^{k} L_{k m}^{l} x^{m}
$$

normalized via

$$
\int_{0}^{\infty} \mathrm{e}^{-x} x^{l+1 / 2} L_{k}^{l+1 / 2}(x) L_{k^{\prime}}^{l+1 / 2}(x) \mathrm{d} x=\lambda_{k}^{l} \delta_{k, k^{\prime}},
$$

with $\lambda_{k}^{l}=(l+k+1 / 2) ! / k !$ and $L_{k m}^{l}=\left[(-1)^{m}(l+k+1 / 2) !\right] /[(k-m) !(l+m+1 / 2) ! m !]$. The $\sigma_{k}^{l}=\sigma_{l} \lambda_{k}^{l}$ term is a normalization factor from the orthogonality relations in (3.17), (3.19).

Finally, the coefficients of the expansion in (3.13) $\boldsymbol{M}_{a}^{l k}$ are

$$
\boldsymbol{M}_{a}^{l k}=\frac{1}{n_{a}} \int \mathrm{d} \boldsymbol{v} f_{a} \frac{L_{k}^{l+1 / 2}\left(c_{a}^{2}\right) \boldsymbol{P}^{l}\left(\boldsymbol{c}_{a}\right)}{\sqrt{\sigma_{k}^{l}}},
$$

which correspond to the moments of $f_{a}$ due to the orthogonality relations in (3.17), (3.19).

By using the expansion in (3.13) in the collision operator in (2.22), a closed form for $C_{a b}$ in terms of products of $\boldsymbol{M}_{a}^{l k}$ can be obtained. For like-species collisions it reads

$$
C_{a a}=\sum_{l k m} \sum_{n q r} \frac{L_{k m}^{l} L_{q r}^{n}}{\sqrt{\sigma_{k}^{l} \sigma_{q}^{n}}} c\left(f_{a}^{l k m}, f_{a}^{n q r}\right),
$$

with

$$
c\left(f_{a}^{l k m}, f_{a}^{n q r}\right)=f_{a M} \sum_{u=0}^{\min (2, l, n)} \nu_{* a a u}^{l m, n r}\left(c_{a}^{2}\right) \sum_{i=0}^{\min (l, n)-u} d_{i}^{l-u, n-u} \boldsymbol{P}^{l+n-2(i+u)}\left(\hat{\boldsymbol{c}_{a}}\right) \cdot \overline{\boldsymbol{M}_{a}^{l k} \cdot{ }^{i+u} \boldsymbol{M}_{a}^{n q}},
$$

where $\hat{\boldsymbol{c}_{a}}=\boldsymbol{c}_{a} / c_{a}, \cdot^{n}$ is the $n$-fold inner product (e.g. for the matrix $\boldsymbol{A}=A_{i j}$, $\left.\left(\boldsymbol{A} \cdot{ }^{1} \boldsymbol{A}\right)_{i j}=\sum_{k} A_{k i} A_{k j}\right)$ and $\overline{\boldsymbol{A}}$ the traceless symmetrization of $\boldsymbol{A}$ (e.g. $\overline{\boldsymbol{A}}=\left(A_{i j}+\right.$ $\left.A_{j i}\right) / 2-\delta_{i j} \sum_{k} A_{k k} / 3$ ). We refer the reader to Ji \& Held (2009) for the explicit form of the $v_{* a b u}^{l m, n r}$ coefficients. The expansion of the unlike-species collisions is reported in Ji \& Held (2008). 


\subsection{Guiding-centre moment expansion of the collision operators}

In order to apply the gyroaveraging operator to the like-species collision operator $C_{a a}$ in (3.21), we expand the fluid moments as $\boldsymbol{M}_{a}^{l k}=\boldsymbol{M}_{a 0}^{l k}+\epsilon \boldsymbol{M}_{a 1}^{l k}+\cdots$, aiming at representing the collision operator up to $O\left(\epsilon_{v} \epsilon\right)$. An analytical expression for the leading order $\boldsymbol{M}_{a 0}^{l k}$ in terms of guiding-centre moments $N_{a}^{p j}$ can be obtained as follows. By splitting $f_{a}=\left\langle f_{a}\right\rangle+\tilde{f}_{a}$ when evaluating the fluid moments $\boldsymbol{M}_{a}^{\text {lk }}$ according to (3.20), we obtain

$$
\boldsymbol{M}_{a}^{l k}=\frac{1}{n_{a}} \int \mathrm{d}^{3} x^{\prime} \mathrm{d}^{3} v^{\prime} \delta\left(\boldsymbol{x}^{\prime}-\boldsymbol{x}\right) \frac{L_{k}^{l+1 / 2}\left(c_{a}^{\prime 2}\right) \boldsymbol{P}^{l}\left(\boldsymbol{c}_{a}^{\prime}\right)}{\sqrt{\sigma_{k}^{l}}}\left(\left\langle f_{a}\right\rangle+\tilde{f}_{a}\right),
$$

where the Dirac delta function was introduced to convert the velocity integral into an $(\boldsymbol{x}, \boldsymbol{v})$ integral that encompasses the full phase space. Since the volume element in phase space can be written as $\mathrm{d}^{3} \boldsymbol{x} \mathrm{d}^{3} \boldsymbol{v}=\left(B_{\|}^{*} / m\right) \mathrm{d} \boldsymbol{R} \mathrm{d} v_{\|} \mathrm{d} \mu \mathrm{d} \theta$ (Cary \& Brizard 2009), and defining $\boldsymbol{x}^{\prime}=\boldsymbol{R}+\rho_{a} \boldsymbol{a}$, we can write the fluid moments in (3.23) as

$$
\boldsymbol{M}_{a}^{l k}=\frac{1}{n_{a}} \int \mathrm{d} \boldsymbol{R} \mathrm{d} v_{\|} \mathrm{d} \mu \mathrm{d} \theta \frac{B_{\|}^{*}}{m_{a}} \delta\left(\boldsymbol{x}-\boldsymbol{R}-\rho_{a} \boldsymbol{a}\right) \frac{L_{k}^{l+1 / 2}\left(c_{a}^{\prime 2}\right) \boldsymbol{P}^{l}\left(\boldsymbol{c}_{a}^{\prime}\right)}{\sqrt{\sigma_{k}^{l}}}\left(\left\langle F_{a}\right\rangle+\tilde{F}_{a}\right),
$$

where $\left\langle f_{a}\right\rangle$ and $\tilde{f}_{a}$ in (3.23) are written in terms of guiding-centre coordinates using (2.29). Neglecting the higher order $\rho_{a}$ and $\tilde{F}_{a}$ terms, the leading-order fluid moments $\boldsymbol{M}_{a 0}^{l k}$ are given by

$$
\boldsymbol{M}_{a 0}^{l k}=\frac{1}{n_{a}} \int \mathrm{d} v_{\|} \mathrm{d} \mu \mathrm{d} \theta \frac{B_{\|}^{*}}{m_{a}} \frac{L_{k}^{l+1 / 2}\left(c_{a}^{\prime 2}\right) \boldsymbol{P}^{l}\left(\boldsymbol{c}_{a}^{\prime}\right)}{\sqrt{\sigma_{k}^{l}}}\left\langle F_{a}\right\rangle .
$$

The $\theta$ integration can be performed by making use of the gyroaveraging formula of the $\boldsymbol{P}^{l}$ tensor (Ji \& Held 2009)

$$
\left\langle\boldsymbol{P}^{l}\left(\boldsymbol{c}_{a}\right)\right\rangle=c_{a}^{l} P_{l}\left(\xi_{a}\right) \boldsymbol{P}^{l}(\boldsymbol{b}),
$$

where $\xi_{a}=\boldsymbol{c}_{a} \cdot \boldsymbol{b} / c_{a}$ is the pitch-angle velocity coordinate, and $P_{l}$ is a Legendre polynomial defined by

$$
P_{l}(x)=\frac{1}{2^{l} l !} \frac{\mathrm{d}^{l}}{\mathrm{~d} x^{l}}\left[\left(x^{2}-1\right)^{l}\right],
$$

and normalized via

$$
\int_{-1}^{1} P_{l}(x) P_{l^{\prime}}(x) \mathrm{d} x=\frac{\delta_{l l^{\prime}}}{l+1 / 2},
$$

yielding

$$
\boldsymbol{M}_{a 0}^{l k}=\frac{\boldsymbol{P}^{l}(\boldsymbol{b})}{n_{a}} \int \mathrm{d} v_{\|} \mathrm{d} \mu \mathrm{d} \theta \frac{B_{\|}^{*}}{m_{a}} \frac{L_{k}^{l+1 / 2}\left(c_{a}^{\prime 2}\right) c_{a}^{l} P_{l}\left(\xi_{a}\right)}{\sqrt{\sigma_{k}^{l}}}\left\langle F_{a}\right\rangle .
$$

Finally, we use the basis transformation

$$
c_{a}^{l} P_{l}\left(\xi_{a}\right) L_{k}^{l+1 / 2}\left(c_{a}^{2}\right)=\sum_{p=0}^{l+2 k} \sum_{j=0}^{k+\lfloor l / 2\rfloor} T_{a l k}^{p j} H_{p}\left(s_{\| a}\right) L_{j}\left(s_{\perp a}^{2}\right),
$$


with the inverse

$$
H_{p}\left(s_{\| a}\right) L_{j}\left(s_{\perp a}^{2}\right)=\sum_{l=0}^{p+2 j} \sum_{k=0}^{j+\lfloor p / 2\rfloor}\left(T_{a}^{-1}\right)_{p j}^{l k} c_{a}^{l} P_{l}\left(\xi_{a}\right) L_{k}^{l+1 / 2}\left(c_{a}^{2}\right),
$$

to obtain an expression in terms of the Hermite-Laguerre basis. A numerical evaluation of $T_{a l k}^{p j}$ and $\left(T_{a}^{-1}\right)_{p j}^{l k}$ was carried out in Omotani et al. (2015). Instead, in appendix A, we derive the analytic expressions of both $T_{a l k}^{p j}$ and $\left(T_{a}^{-1}\right)_{p j}^{l k}$.

Using the definition of guiding-centre moments $N_{a}^{p j}$ in (3.6), the leading-order fluid moment $\boldsymbol{M}_{a 0}^{l k}$ is then given by

$$
n_{a} \boldsymbol{M}_{a 0}^{l k}=N_{a} \boldsymbol{P}^{l}(\boldsymbol{b}) \mathcal{N}_{a}^{l k}
$$

where we define

$$
\mathcal{N}_{a}^{l k}=\sum_{p=0}^{l+2 k} \sum_{j=0}^{k+\lfloor l / 2\rfloor} T_{a l k}^{p j} N_{a}^{p j} \sqrt{\frac{2^{p} p !}{\sigma_{k}^{l}}} .
$$

The leading-order part $C_{a a 0}$ of the collision operator $C_{a a}$ can be calculated by approximating $\boldsymbol{M}_{a}^{l k}$ appearing in (3.22) with $\boldsymbol{M}_{a 0}^{l k}$. For the ions, the largest contribution to $\boldsymbol{M}_{i}^{l k}-\boldsymbol{M}_{i 0}^{l k}$ is of order $\epsilon$ and it is given by the $\rho_{i}$ appearing in (3.24) (the $\tilde{F}_{i}$ correction is smaller since $\tilde{F}_{i} \lesssim \epsilon^{2}\left\langle F_{i}\right\rangle$, see (2.35)). Therefore, by using the ordering in (2.28), the largest correction to $C_{i i 0}$ is $O\left(\sqrt{m_{e} / m_{i}} \epsilon \epsilon_{v}\right)$. The correction to $C_{e e 0}$ is of the same order. It follows that we can approximate $C_{a a}$ appearing in (3.22) with $C_{a a 0}$ to represent the collision operator up to $O\left(\epsilon_{v} \epsilon\right)$.

As an aside, we note that the relationship between the guiding-centre and fluid moments in (3.32) provides, for the indices $(l, k)=(0,0)$,

$$
n_{a}=N_{a}
$$

while, for $(l, k)=(0,1)$,

$$
T_{a}=\frac{T_{\| a}+2 T_{\perp a}}{3} .
$$

Moreover, the $(l, k)=(2,0)$ moment provides a relationship useful to express the viscosity tensor $\boldsymbol{\Pi}_{a}=\int\left(\boldsymbol{c}_{a} \boldsymbol{c}_{a}-c_{a}^{2} I\right) f_{a} \mathrm{~d} \boldsymbol{v}$

$$
\boldsymbol{\Pi}_{a}=\boldsymbol{b} \boldsymbol{b} N\left(T_{\| a}-T_{\perp a}\right)
$$

and $(l, k)=(1,1)$ gives

$$
\boldsymbol{q}_{a}=\left(\frac{Q_{\| a}}{2}+Q_{\perp a}\right) \boldsymbol{b}
$$

with $\boldsymbol{q}_{a}$ the heat flux density $\boldsymbol{q}_{a}=m \int \boldsymbol{c}_{a} c_{a}^{2} f_{a} \mathrm{~d} \boldsymbol{v} / 2$.

In order to express the Boltzmann equation, (2.32), in terms of the guiding-centre moments $N_{a}^{p j}$, we evaluate the guiding-centre moments of $\left\langle C_{a a}\right\rangle$, namely

$$
C_{a a}^{p j}=\frac{1}{N_{a}} \int\left\langle C_{a a 0}\right\rangle \frac{H_{p}\left(s_{\| a}\right) L_{j}\left(s_{\perp a}^{2}\right)}{\sqrt{2^{p} p !}} \frac{B}{m_{a}} \mathrm{~d} v_{\|} \mathrm{d} \mu \mathrm{d} \theta .
$$


By using the gyroaveraging property (3.26) of $\boldsymbol{P}^{l}\left(\boldsymbol{c}_{a}\right)$ in the like-species operator in (3.21), (3.22) (with $\boldsymbol{M}_{a}^{l k}=\boldsymbol{M}_{a 0}^{l k}$ ), and the relation (3.32) between $\boldsymbol{M}_{a 0}^{l k}$ and $N_{a}^{p j}$, the gyroaveraged collision operator coefficients $\left\langle c\left(f_{a}^{l k m}, f_{a}^{n q r}\right)\right\rangle$ are given by

$$
\left\langle c\left(f_{a}^{l k m}, f_{a}^{n q r}\right)\right\rangle=f_{a M} \sum_{u=0}^{\min (2, l, n)} v_{* a a u}^{l m, n r}\left(c_{a}^{2}\right) \sum_{i=0}^{\min (l, n)-u} d_{i}^{l-u, n-u} P_{l+n-2(i+u)}(\xi) \mathcal{N}_{a}^{l k} \mathcal{N}_{a}^{n q} \mathcal{P}_{i+u}^{l, n},
$$

with $\mathcal{P}_{i+u}^{l, n}=\boldsymbol{P}^{l+n-2(i+u)} \cdot \overline{\boldsymbol{P}^{l} \cdot{ }^{i+u} \boldsymbol{P}^{n}}$.

Using the basis transformation of (3.31) to express $H_{p}\left(s_{\| a}\right) L_{j}\left(s_{\perp a}^{2}\right)$ in (3.38) in terms of $c_{a}^{l} P_{l}\left(\xi_{a}\right) L_{k}^{l+1 / 2}\left(c_{a}^{2}\right)$, and performing the resulting integral, we obtain

$$
\begin{aligned}
C_{a a}^{p j}= & \sum_{l, k} \sum_{n, q} \sum_{u=0}^{\min (2, l, n)} \sum_{i=0}^{\min (l, n)-u} \sum_{e=0}^{p+2 j} \sum_{f=0}^{j+\lfloor p / 2\rfloor} \sum_{g=0}^{f} \sum_{m=0}^{k} \sum_{r=0}^{q} \\
& \times \frac{L_{k m}^{l} L_{q r}^{n} L_{f g}^{e} d_{i}^{l-u, n-u}}{\sqrt{\sigma_{k}^{l} \sigma_{q}^{n}}(e+1 / 2) 4 \pi} \frac{C_{* a a u}^{e g, l m, n r}}{\sqrt{2^{p} p !}} \delta_{e, l+n-2(i+u)}\left(T^{-1}\right)_{p j}^{e f} \mathcal{N}_{a}^{l k} \mathcal{N}_{a}^{n q} \mathcal{P}_{i+u}^{l, n},
\end{aligned}
$$

with $C_{* a a b u}^{j w, l m, n r}=\int \mathrm{d} v c_{a}^{2 w+j} f_{M a} v_{* a a u}^{l m, n r}$.

We now turn to the electron-ion collision operator, $C_{e i}=C_{e i}^{0}+C_{e i}^{1}$, with $C_{e i}^{0}$ given by (2.25) and $C_{e i}^{1}$ given by (2.26). As the basis $L_{k}^{l+1 / 2} \boldsymbol{P}^{l}\left(\boldsymbol{c}_{a}\right)$ is an eigenfunction of the Lorentz pitch-angle scattering operator $C_{e i}^{0}$ with eigenvalue $-l(l+1)$ (Ji \& Held 2008), we write $C_{e i}^{0}$ as

$$
C_{e i}^{0}=-\sum_{l, k} \frac{n_{i} L_{e i}}{8 \pi c_{e}^{3}} \frac{l(l+1) f_{e M}}{\sqrt{\sigma_{k}^{l}}} L_{k}^{l+1 / 2}\left(c_{e}^{2}\right) \boldsymbol{P}^{l}\left(\boldsymbol{c}_{e}\right) \cdot \boldsymbol{M}_{e}^{l k}
$$

Similarly to like-species collisions, we approximate $\boldsymbol{M}_{e}^{l k} \simeq \boldsymbol{M}_{e 0}^{l k}$ in (3.41), representing $C_{e i}^{0}$ accurately up to $O\left(\epsilon_{v} \epsilon\right)$. Using the basis transformation in (3.31) and the gyroaverage property of $\boldsymbol{P}^{l}\left(\boldsymbol{c}_{a}\right)$ in (3.26), we take guiding-centre moments of $C_{e i}$ of the form (3.38), and obtain

$$
C_{e i}^{p j}=-\frac{v_{e i}}{8 \pi^{3 / 2}} \sum_{l=0}^{p+2 j} \sum_{f=0}^{j+\lfloor p / 2\rfloor} \frac{\left(T_{e}^{-1}\right)_{p j}^{l f}}{\sqrt{2^{p} p !}}\left[\sum_{k=0}^{\infty} A_{e i}^{l f, k} \mathcal{N}_{e}^{l k}-\delta_{l, 1} \frac{u_{\| i}-u_{\| e}}{v_{t h e}} \frac{8}{3} \frac{\Gamma(f+3 / 2)}{f ! \sqrt{\pi}}\right],
$$

where the $A_{e i}$ coefficients are given by

$$
A_{e i, 0}^{l f, k}=\frac{l(l+1)}{l+1 / 2}\left|\boldsymbol{P}^{l}(\boldsymbol{b})\right|^{2} \sum_{m=0}^{f} \sum_{n=0}^{k} \frac{L_{f m}^{l} L_{k n}^{l}}{\sqrt{\sigma_{k}^{l}}}(l+m+n-1) ! .
$$

Finally, for the ion-electron collision operator, $C_{i e}$, we neglect $O\left(\sqrt{m_{e} / m_{i}} \epsilon_{v} \epsilon\right)$ corrections by approximating $F_{i} \simeq\left\langle F_{i}\right\rangle$, and use the transformation in (2.3) to convert the $C_{i e}$ operator in (2.27) to guiding-centre variables, yielding 


$$
\begin{aligned}
C_{i e}= & \frac{\boldsymbol{R}_{e i}}{m_{i} n_{i} v_{t h i}} \cdot\left[\boldsymbol{c}_{\perp} \frac{m_{i} v_{t h i}^{2}}{B} \frac{\partial\left\langle F_{i}\right\rangle}{\partial \mu}+\boldsymbol{b} \frac{\partial\left\langle F_{i}\right\rangle}{\partial c_{\| i}}\right]+v_{e i} \frac{m_{e}}{m_{i}} \frac{n_{e}}{n_{i}} \\
& \times\left[3\left\langle F_{i}\right\rangle+c_{\| i} \frac{\partial\left\langle F_{i}\right\rangle}{\partial c_{\| i}}+2 \mu \frac{\partial\left\langle F_{i}\right\rangle}{\partial \mu}+\frac{T_{e}}{2 T_{i}} \frac{\partial^{2}\left\langle F_{i}\right\rangle}{\partial c_{\| i}^{2}}+\frac{2 T_{e}}{B} \frac{\partial}{\partial \mu}\left(\mu \frac{\partial\left\langle F_{i}\right\rangle}{\partial \mu}\right)\right] .
\end{aligned}
$$

By evaluating $\boldsymbol{R}_{e i}$ at the guiding-centre position $\boldsymbol{R}$, we write $\boldsymbol{R}_{e i} \cdot \boldsymbol{b}=N_{e} m_{e} v_{t h \| e} C_{e i}^{10} /$ $\sqrt{2}+O\left(\sqrt{m_{e} / m_{i}} \epsilon_{\nu} \epsilon\right)$ and gyroaverage (3.44), yielding

$$
\begin{aligned}
\left\langle C_{i e}\right\rangle= & \frac{C_{e i}^{10}}{\sqrt{2}} \frac{m_{e}}{m_{i}} \frac{N_{e}}{n_{i}} \frac{v_{t h \| e}}{v_{t h \| i}} \frac{\partial\left\langle F_{i}\right\rangle}{\partial s_{\|}}+v_{e i} \frac{m_{e}}{m_{i}} \frac{n_{e}}{n_{i}} \\
& \times\left[3\left\langle F_{i}\right\rangle+s_{\| i} \frac{\partial\left\langle F_{i}\right\rangle}{\partial s_{\| i}}+2 \mu \frac{\partial\left\langle F_{i}\right\rangle}{\partial \mu}+\frac{T_{e}}{2 T_{\| i}} \frac{\partial^{2}\left\langle F_{i}\right\rangle}{\partial s_{\| i}^{2}}+\frac{2 T_{e}}{B} \frac{\partial}{\partial \mu}\left(\mu \frac{\partial\left\langle F_{i}\right\rangle}{\partial \mu}\right)\right],
\end{aligned}
$$

where we used $c_{\| i}^{2}=s_{\| i}^{2} T_{\| i} / T_{i}$. Taking guiding-centre moments of the form (3.38) of $\left\langle C_{i e}\right\rangle$ in (3.45), we obtain

$$
C_{i e}^{p j}=v_{e i} \frac{m_{e}}{m_{i}} \sum_{l k} B_{l k}^{p j} N_{i}^{l k}
$$

with

$$
\begin{aligned}
B_{l k}^{p j}= & 2 j \delta_{l p} \delta_{k j-1}\left(1-\frac{T_{e}}{T_{\perp i}}\right)-\sqrt{p} \frac{v_{t h \| e}}{v_{t h \| i}} \frac{C_{e i}^{10}}{v_{e i}} \delta_{l p-1} \delta_{k j} \\
& -(p+2 j) \delta_{l p} \delta_{k j}+\sqrt{p(p-1)} \delta_{l p-2} \delta_{k j}\left(\frac{T_{e}}{T_{\| i}}-1\right) .
\end{aligned}
$$

\section{Moment hierarchy}

In this section, we derive a set of equations that describe the evolution of the guiding-centre moments $N_{a}^{p j}$, by integrating in guiding-centre velocity space the conservative form of the Boltzmann equation, (2.32), with the weights $H_{p}\left(s_{\| a}\right) L_{j}\left(s_{\perp a}^{2}\right)$. First, we highlight the dependence of $\dot{\boldsymbol{R}}$ and $\dot{v}_{\|}$on $s_{\| a}$ and $s_{\perp a}^{2}$ by rewriting the equations of motion as

$$
\dot{\boldsymbol{R}}=\boldsymbol{U}_{0 a}+\boldsymbol{U}_{p a}^{*}+s_{\perp a}^{2} \boldsymbol{U}_{\nabla B a}^{*}+s_{\| a}^{2} \boldsymbol{U}_{k a}^{*}+s_{\| a}\left(v_{t h \| a} \boldsymbol{b}+\boldsymbol{U}_{p a}^{* t h}\right),
$$

and

$$
m_{a} \dot{v}_{\|}=F_{\| a}-s_{\perp a}^{2} F_{M a}+s_{\| a} F_{p a}^{t h}-m_{a} \mathcal{A} .
$$

In (4.1), (4.2), $\boldsymbol{U}_{0 a}=\boldsymbol{v}_{E}+\boldsymbol{u}_{\| a} \boldsymbol{b}$ is the lowest-order guiding-centre fluid velocity, $\boldsymbol{U}_{\nabla B a}^{*}=\left(T_{\perp a} / m_{a}\right)\left(\boldsymbol{b} \times \nabla B / \Omega_{a}^{*} B\right)$ is the fluid grad-B drift, with $\Omega_{a}^{*}=q_{a} B_{\|}^{*} / m_{a}, \boldsymbol{U}_{k a}^{*}=$ $\left(2 T_{\| a} / m_{a}\right)\left(\boldsymbol{b} \times \boldsymbol{k} / \Omega_{a}^{*}\right)$ is the fluid curvature drift with $\boldsymbol{k}=\boldsymbol{b} \cdot \nabla \boldsymbol{b}, \boldsymbol{U}_{p a}^{*}=\left(\boldsymbol{b} / \Omega_{a}^{*}\right) \times$ $\mathrm{d}_{0} U_{0 a} / \mathrm{d} t$ is the fluid polarization drift, $F_{\| a}=q_{a} E_{\|}+m_{a} \boldsymbol{v}_{E} \cdot \mathrm{d}_{0} \boldsymbol{b} / \mathrm{d} t, F_{M a}=T_{\perp a} \nabla_{\|} \ln B$ is the mirror force, and both $\boldsymbol{U}_{p a}^{* t h}$ and $F_{p a}^{t h}$ are related to gradients of the electromagnetic fields

$$
\boldsymbol{U}_{p a}^{* t h}=v_{t h \| a} \frac{\boldsymbol{b}}{\Omega_{a}^{*}} \times\left(\boldsymbol{b} \cdot \nabla \boldsymbol{v}_{E}+\boldsymbol{v}_{E} \cdot \nabla \boldsymbol{b}+2 u_{\| a} \boldsymbol{k}\right)
$$




$$
F_{p a}^{t h}=m_{a} v_{t h \| a} \boldsymbol{b} \cdot\left(\frac{\boldsymbol{k} \times \boldsymbol{E}}{B}\right) .
$$

The fluid-convective derivative operator is defined as

$$
\frac{\mathrm{d}_{0 a}}{\mathrm{~d} t}=\partial_{t}+\boldsymbol{U}_{0 a} \cdot \nabla
$$

Next, to obtain an equation for the moment $N_{a}^{p j}$, we apply the guiding-centre moment operator

$$
\begin{aligned}
\|\chi\|_{a}^{* p j} & =\frac{1}{N_{a} B}\left\|\chi H_{p}\left(s_{\| a}\right) L_{j}\left(s_{\perp a}^{2}\right) B_{\|}^{*}\right\| \\
& =\frac{1}{N_{a}} \int \chi \frac{B_{\|}^{*}}{m_{a}}\left\langle F_{a}\right\rangle \frac{H_{p}\left(s_{\| a}\right) L_{j}\left(s_{\perp a}^{2}\right)}{\sqrt{2^{p} p !}} \mathrm{d} v_{\|} \mathrm{d} \mu \mathrm{d} \theta,
\end{aligned}
$$

to Boltzmann's equation, (2.32). By defining $\|1\|_{a}^{* p j}=\bar{N}_{a}^{p j}$ such that

$$
\begin{aligned}
\bar{N}_{a}^{p j}= & N_{a}^{p j}\left(1+\frac{\boldsymbol{b} \cdot \boldsymbol{\nabla} \times \boldsymbol{v}_{E}}{\Omega_{a}}+u_{\| a} \frac{\boldsymbol{b} \cdot \boldsymbol{\nabla} \times \boldsymbol{b}}{\Omega_{a}}\right) \\
& +v_{t h \| a} \frac{\boldsymbol{b} \cdot \boldsymbol{\nabla} \times \boldsymbol{b}}{\sqrt{2} \Omega_{a}}\left(\sqrt{p+1} N_{a}^{p+1 j}+\sqrt{p} N_{a}^{p-1 j}\right),
\end{aligned}
$$

and

$$
\frac{\mathrm{d}_{a}^{* p j}}{\mathrm{~d} t}=\bar{N}_{a}^{p j} \frac{\partial}{\partial t}+\|\dot{\boldsymbol{R}}\|_{a}^{* p j} \cdot \nabla,
$$

the drift-kinetic moment hierarchy conservation equation for species $a$ is

$$
\frac{\partial \bar{N}_{a}^{p j}}{\partial t}+\nabla \cdot\|\dot{\boldsymbol{R}}\|_{a}^{* p j}-\frac{\sqrt{2 p}}{v_{t h \| a}}\left\|\dot{v}_{\|}\right\|_{a}^{* p-1 j}+\mathcal{F}_{a}^{p j}=\sum_{b} C_{a b}^{p j},
$$

where we identify the fluid operator

$$
\begin{aligned}
\mathcal{F}_{a}^{p j}= & \frac{\mathrm{d}_{a}^{* p j}}{\mathrm{~d} t} \ln \left(N_{a} T_{\| a}^{p / 2} T_{\perp a}^{j} B^{-j}\right)+\frac{\sqrt{2 p}}{v_{t h \| a}} \frac{\mathrm{d}^{* p-1 j} u_{\| a}}{\mathrm{~d} t} \\
& +\frac{\sqrt{p(p-1)}}{2} \frac{\mathrm{d}_{a}^{* p-2 j}}{\mathrm{~d} t} \ln T_{\| a}-j \frac{\mathrm{d}_{a}^{* p j-1}}{\mathrm{~d} t} \ln \left(\frac{T_{\perp a}}{B}\right),
\end{aligned}
$$

since it is the key item that describes the evolution of the guiding-centre fluid properties $N_{a}, u_{\| a}, P_{\perp a}$, and $P_{\| a}$ (see (6)).

The guiding-centre moments of the particle's equations of motion are given by

$$
\begin{aligned}
\|\dot{\boldsymbol{R}}\|_{a}^{* p j}= & \sum_{l, k}\left(\boldsymbol{U}_{0 a} \delta_{p l} \delta_{j k}+v_{t h \| a} \boldsymbol{b} \mathcal{V}_{l k}^{1 p j}\right) \bar{N}_{a}^{l k} \\
& +\left(\boldsymbol{U}_{p a} \delta_{p l} \delta_{j k}+\boldsymbol{U}_{p a}^{t h} \mathcal{V}_{l k}^{1 p j}+\boldsymbol{U}_{\nabla B a} \mathcal{M}_{l k}^{p j}+\boldsymbol{U}_{k a} \mathcal{V}_{l k}^{2 p j}\right) N_{a}^{l k}, \\
m_{a}\left\|\dot{v}_{\|}\right\|_{a}^{* p j}= & \sum_{l, k}\left[F_{\| a} \delta_{p, l} \delta_{j, k}+F_{p a}^{t h} \mathcal{V}_{l k}^{1 p j}+F_{M a} \mathcal{M}_{l k}^{p j}\right] \bar{N}_{a}^{l k}+m_{a}\|\mathcal{A}\|_{a}^{* p j},
\end{aligned}
$$


where the phase-mixing operators read

$$
\begin{gathered}
\mathcal{V}_{l k}^{1 p j}=\left(\sqrt{\frac{p+1}{2}} \delta_{p+1, l}+\sqrt{\frac{p}{2}} \delta_{p-1, l}\right) \delta_{k, j}, \\
\mathcal{V}_{l k}^{2 p j}=\left[\delta_{p, l}\left(p+\frac{1}{2}\right)+\frac{\sqrt{(p+2)(p+1)}}{2} \delta_{p+2, l}+\frac{\sqrt{p(p-1)}}{2} \delta_{p-2, l}\right] \delta_{j, k}, \\
\mathcal{M}_{l k}^{p j}=(2 j+1) \delta_{p, l} \delta_{j, k}-(j+1) \delta_{p, l} \delta_{j+1, k}-j \delta_{p, l} \delta_{j-1, k} .
\end{gathered}
$$

The expressions of $\boldsymbol{U}_{p a}, U_{\nabla B_{a}}, U_{p a}^{t h}$ and $\boldsymbol{U}_{k a}$ are derived from $\boldsymbol{U}_{p a}^{*}, U_{\nabla B a}^{*}, U_{p a}^{* t h}$ and $\boldsymbol{U}_{k a}^{*}$ by replacing $\Omega_{a}^{*}$ with $\Omega_{a}$. The expression of $\|\mathcal{A}\|^{* p j}$ can be found in appendix $\mathrm{B}$.

Similar moment hierarchy models (with uniform magnetic fields) have been numerically implemented, and successfully compared with their kinetic counterpart (Paškauskas \& De Ninno 2009; Loureiro et al. 2016; Schekochihin et al. 2016; Grošelj et al. 2017), and even shown to be more efficient than other velocity discretization techniques in the same region of validity (Camporeale et al. 2016). Equation (4.9) generalizes such models to spatially varying fields and full Coulomb collisions, while retaining phase-mixing operators that couple nearby Hermite and Laguerre moments and providing a close form for the projection of the Coulomb operator in velocity space. We also note that the use of shifted velocity polynomials in the Hermite-Laguerre basis, which gives rise to the fluid operator $\mathcal{F}_{a}^{p j}$, allows us to have an efficient representation of the distribution function both in the weak $\left(u_{\| a} \ll v_{t h a}\right)$ and strong flow $\left(u_{\| a} \sim v_{\text {tha }}\right)$ regimes. As we will see in $\S 6$, the fluid operator $\mathcal{F}_{a}^{p j}$ generates the lowest-order fluid equations, as it is present even if all kinetic moments $N_{a}^{p j}$ (except $N_{a}^{00}$ ) are set to zero.

\section{Poisson's equation}

We use Poisson's equation to evaluate the electric field appearing in the moment hierarchy equation, equation (4.9). In $(\boldsymbol{x}, \boldsymbol{v})$ coordinates, Poisson's equation reads

$$
\epsilon_{0} \nabla \cdot \boldsymbol{E}=\sum_{a} q_{a} n_{a}=\sum_{a} q_{a} \int f_{a} \mathrm{~d}^{3} v
$$

Following the same steps used to derive (3.24) from (3.20), we can write Poisson's equation, equation (5.1), as

$$
\epsilon_{0} \nabla \cdot \boldsymbol{E}=\sum_{a} q_{a} \int \mathrm{d}^{3} \boldsymbol{R} \mathrm{d} v_{\|} \mathrm{d} \mu \mathrm{d} \theta \frac{B_{\|}^{*}}{m} \delta\left(\boldsymbol{R}+\rho_{a} \boldsymbol{a}-\boldsymbol{x}\right) F_{a}\left(\boldsymbol{R}, v_{\|}, \mu, \theta\right)
$$

Equation (5.2) shows that all particles that have a Larmor orbit crossing a given point $\boldsymbol{x}$, give a contribution to the charge density at this location.

Performing the integral over $\boldsymbol{R}$ and introducing the Fourier transform $F_{a}\left(\boldsymbol{x}-\rho_{a} \boldsymbol{a}, \boldsymbol{v}_{\|}, \mu, \theta\right)=\int \mathrm{d}^{3} \boldsymbol{k} F_{a}\left(\boldsymbol{k}, \boldsymbol{v}_{\|}, \mu, \theta\right) \mathrm{e}^{-\mathrm{i} \boldsymbol{k} \cdot \boldsymbol{x}} \mathrm{e}^{\mathrm{i} \rho_{a} \boldsymbol{k} \cdot \boldsymbol{a}}$, equation (5.2) can be rewritten as

$$
\epsilon_{0} \nabla \cdot \boldsymbol{E}=\sum_{a} q_{a} \int \mathrm{d} v_{\|} \mathrm{d} \mu \mathrm{d}^{3} \boldsymbol{k} \mathrm{d} \theta \frac{B_{\|}^{*}}{m_{a}} F_{a}\left(\boldsymbol{k}, v_{\|}, \mu, \theta\right) \mathrm{e}^{-\mathrm{i} \boldsymbol{k} \cdot \boldsymbol{x}} \mathrm{e}^{\mathrm{i} \rho_{a} \boldsymbol{k} \cdot \boldsymbol{a}} .
$$


To perform the $\boldsymbol{k}$ integration, we use the cylindrical coordinate system $\left(k_{\perp}, \alpha, k_{\|}\right)$, expressing $\boldsymbol{k}=k_{\perp}\left(\cos \alpha \boldsymbol{e}_{1}+\sin \alpha \boldsymbol{e}_{2}\right)+k_{\|} \boldsymbol{b}$, such that $\boldsymbol{k} \cdot \boldsymbol{a}=k_{\perp} \cos (\theta-\alpha)$. This coordinate system allows us to express $\mathrm{e}^{\mathrm{i} \rho_{a} \boldsymbol{k} \cdot \boldsymbol{a}}$ in (5.3) in terms of Bessel functions. Indeed, $\mathrm{e}^{\mathrm{i} k_{\perp} \rho_{a} \cos (\theta-\alpha)}=J_{0}\left(k_{\perp} \rho_{a}\right)+2 \sum_{l=1}^{\infty} J_{l}\left(k_{\perp} \rho_{a}\right) \mathrm{i}^{l} \cos [l(\theta-\alpha)]$ (Abramowitz, Stegun $\&$ Miller 1965), where $J_{l}\left(k_{\perp} \rho_{a}\right)$ is the Bessel function of the first kind of order $l$. We can then write

$$
\epsilon_{0} \nabla \cdot \boldsymbol{E}=\sum_{a} q_{a} \int \mathrm{d} v_{\|} \mathrm{d} \mu \mathrm{d} \theta \frac{B_{\|}^{*}}{m_{a}}\left(\Gamma_{0}\left[F_{a}\right]+2 \sum_{l=1}^{\infty} i^{l} \Gamma_{l}\left[F_{a} \cos [l(\theta-\alpha)]\right]\right)
$$

where the Fourier-Bessel operator $\Gamma_{l}[f]$ is defined as

$$
\Gamma_{l}\left[F_{a}\left(\boldsymbol{k}, v_{\|}, \mu, \theta\right)\right] \equiv \int \mathrm{d}^{3} \boldsymbol{k} J_{l}\left(k_{\perp} \rho_{a}\right) F_{a}\left(\boldsymbol{k}, v_{\|}, \mu, \theta\right) \mathrm{e}^{-\mathrm{i} \boldsymbol{k} \cdot \boldsymbol{x}}
$$

Introducing the Fourier decomposition of $\tilde{F}_{a}$, equation (2.39), in (5.4), we obtain

$$
\epsilon_{0} \nabla \cdot \boldsymbol{E}=\sum_{a} q_{a} \int \mathrm{d} v_{\|} \mathrm{d} \mu \frac{B_{\|}^{*}}{m}\left[\Gamma_{0}\left[\left\langle F_{a}\right\rangle\right]+2 \pi \sum_{l=1}^{\infty} \frac{\mathrm{i}^{l-1}}{l \Omega_{a}} \Gamma_{l}\left[C_{l a} \mathrm{e}^{\mathrm{i} l \alpha}+C_{-l a} \mathrm{e}^{-\mathrm{i} l \alpha}\right]\right],
$$

where the $\theta$ integration was performed by using the identity $\int_{0}^{2 \pi} \mathrm{e}^{\mathrm{i} \theta(l-m)} \mathrm{d} \theta=$ $2 \pi \delta(l-m)$. Notice that $\int_{0}^{2 \pi} \Gamma_{0}\left[F_{a}\right] \mathrm{d} \theta / 2 \pi=\Gamma_{0}\left(\left\langle F_{a}\right\rangle\right)$, and corresponds to the $J_{0}\left(k_{\perp} \rho_{a}\right)$ operator used in most gyrofluid closures (Hammett, Dorland \& Perkins 1992; Snyder \& Hammett 2001; Madsen 2013), and in the gyrokinetic Poisson equation (Dubin, Krommes \& Oberman 1983; Lee 1983).

We now order the terms appearing in (5.6). Using the Taylor series expansion of a Bessel function $J_{l}(x)$ of order $l$ (Abramowitz et al. 1965), we find

$$
\Gamma_{0}\left[\left\langle F_{a}\right\rangle\right] \sim\left[1-\frac{\left(k_{\perp} \rho_{a}\right)^{2}}{4}+O\left(\epsilon^{4}\right)\right]\left\langle F_{a}\right\rangle
$$

while using the orderings of $v_{e}$ and $v_{i}$ in (1.2) and (2.28)

$$
\frac{\Gamma_{l}\left[C_{l a}\right]}{\Omega_{a}} \lesssim \epsilon_{\nu} \epsilon^{l+1}\left\langle F_{a}\right\rangle
$$

for $l \geqslant 1$.

Consistently with $\S 3.3$, we neglect the $l \geqslant 1$ collisional terms, therefore representing Poisson's equation up to $O\left(\epsilon_{\nu} \epsilon\right)$. For the derivation of an higher-order Poisson equation, the treatment of finite $l \geqslant 1$ collisional effects are presented in appendix C. Taylor expanding $J_{0}(x) \simeq 1-x^{2} / 4$, Poisson's equation reads

$$
\epsilon_{0} \nabla \cdot \boldsymbol{E}=\sum_{a} q_{a}\left[N_{a}\left(1+\frac{\boldsymbol{b} \cdot \boldsymbol{\nabla} \times \boldsymbol{b}}{\Omega_{a}} u_{\| a}+\frac{\boldsymbol{b} \cdot \boldsymbol{\nabla} \times \boldsymbol{v}_{E}}{\Omega_{a}}\right)+\frac{1}{2 m_{a}} \nabla_{\perp}^{2}\left(\frac{P_{\perp a}}{\Omega_{a}^{2}}\right)\right] .
$$




\section{Collisional drift-reduced fluid model}

The infinite set of equations that describe the evolution of the moments of the distribution function, equation (4.9), and Poisson's equation, equation (5.9), constitute the drift-reduced model, which is valid for distribution functions arbitrarily far from equilibrium. For practical purposes, a closure scheme must be provided in order to reduce the model to a finite number of equations. In this section, we derive a closure in the high-collisionality regime. For this purpose, we first state in $\S 6.1$ the evolution equations for the fluid moments (i.e. $n_{a}, u_{\| a}, T_{\| a}, T_{\perp a}, Q_{\| a}$ and $Q_{\perp a}$ ), that correspond to the lowest-order indices of the moment hierarchy equation. Then, in $\S 6.2$, we apply a prescription for the higher-order parallel and perpendicular moment equations that allows a collisional closure for $Q_{\| a}$ and $Q_{\perp a}$ in terms of $n_{a}, u_{\| a}, T_{\| a}$ and $T_{\perp a}$. The nonlinear closure prescription used here, sometimes called semi-collisional closure (Zocco \& Schekochihin 2011), can be employed at arbitrary collisionalities by including a sufficiently high number of moments (indeed, it was used in Loureiro et al. (2016) to consider low-collisionality regimes). It also allows us to retain the nonlinear collision contributions inherent to a full-F description that may have the same size as its linear contributions, as pointed out in Catto \& Simakov (2004).

\subsection{Fluid equations}

We first look at the $(p, j)=(0,0)$ case of (4.9). Noting that $\bar{N}_{a}^{00}=0$ and $C_{a b}^{00}=0$, we obtain

$$
\nabla \cdot\|\dot{\boldsymbol{R}}\|_{a}^{* 00}+\mathcal{F}_{a}^{00}=0 .
$$

Evaluating $\|\dot{\boldsymbol{R}}\|_{a}^{* p j}$ in (4.11) and $\mathcal{F}_{a}^{p j}$ in (4.10), for $(p, j)=(0,0)$, equation (6.1) yields the continuity equation

$$
\frac{\mathrm{d}_{a}^{0} N_{a}}{\mathrm{~d} t}+\frac{\mathrm{d}_{0 a}}{\mathrm{~d} t}\left(\frac{N_{a} \nabla_{\perp}^{2} \phi}{\Omega_{a} B}\right)=-N_{a} \nabla \cdot \boldsymbol{u}_{0 a}-\frac{N_{a} \nabla_{\perp}^{2} \phi}{\Omega_{a} B} \nabla \cdot \boldsymbol{U}_{0 a} .
$$

The upper-convective derivative $\mathrm{d}_{a}^{0} / \mathrm{d} t$, defined by

$$
\frac{\mathrm{d}_{a}^{0}}{\mathrm{~d} t}=\frac{\partial}{\partial t}+\boldsymbol{u}_{0 a} \cdot \nabla
$$

is related to the guiding-centre fluid velocity $\boldsymbol{u}_{0 a}$

$$
\boldsymbol{u}_{0 a}=\boldsymbol{U}_{0 a}+\frac{T_{\| a}+T_{\perp a}}{m_{a}} \frac{\boldsymbol{b} \times \nabla B}{\Omega_{a} B}+\frac{\boldsymbol{b}}{\Omega_{a}} \times \frac{\mathrm{d}_{0 a} \boldsymbol{U}_{0 a}}{\mathrm{~d} t},
$$

and it differs from the lower-convective derivative $\mathrm{d}_{0 a} / \mathrm{d} t$ in (4.5) by the addition of the last two terms in (6.4). The vorticity $\nabla_{\perp}^{2} \phi$ is related to the $\boldsymbol{E} \times \boldsymbol{B}$ drift by

$$
\frac{\boldsymbol{b} \cdot \boldsymbol{\nabla} \times \boldsymbol{v}_{E}}{\Omega_{a}}=\frac{\nabla_{\perp}^{2} \phi}{B \Omega_{a}}+O\left(\epsilon^{3}\right)
$$

and it appears in (6.2) due to the difference between $\bar{N}_{a}^{00}$ and $N_{a}^{00}$ [see (4.7)]. To derive (6.2), we use the low- $\beta$ limit expression for $\boldsymbol{b} \times \boldsymbol{k} \simeq(\boldsymbol{b} \times \nabla B) / B$ and 
neglect $u_{\| a} \boldsymbol{b} \cdot \nabla \times \boldsymbol{b} / \Omega_{a}$ as

$$
\frac{u_{\| a} \boldsymbol{b} \cdot \nabla \times \boldsymbol{b}}{\Omega_{a}} \sim \frac{T_{e}}{T_{i}} \beta \sim \epsilon^{3},
$$

therefore keeping up to $O\left(\epsilon^{2}\right)$ terms (namely the $\nabla_{\perp}^{2} \phi$ term in (6.5)).

The parallel momentum equation is obtained by setting $(p, j)=(1,0)$ in $(4.9)$, yielding

$$
\begin{aligned}
m_{a} \frac{\mathrm{d}_{a}^{0} u_{\| a}}{\mathrm{~d} t}= & \frac{m_{a} v_{t h \| a}}{\sqrt{2}} \sum_{b} C_{a b}^{10}-\frac{m_{a} \nabla_{\perp}^{2} \phi}{\Omega_{a} B} \frac{\mathrm{d}_{0} u_{\| a}}{\mathrm{~d} t}-\frac{m_{a}}{\sqrt{2} N_{a}} \nabla \cdot\left(\boldsymbol{u}_{a}^{1} N_{a} v_{t h \| a}\right) \\
& +m_{a}\|\mathcal{A}\|_{a}^{* 00}+\left(1+\frac{\nabla_{\perp}^{2} \phi}{\Omega_{a} B}\right)\left(q_{a} E_{\|}-T_{\perp a} \frac{\nabla_{\|} B}{B}+m_{a} \boldsymbol{v}_{E} \cdot \frac{\mathrm{d}_{0 a} \boldsymbol{b}}{\mathrm{d} t}\right)
\end{aligned}
$$

with

$$
\boldsymbol{u}_{a}^{1}=\frac{\boldsymbol{U}_{p a}^{t h}}{\sqrt{2}}+\frac{\sqrt{2}}{m_{a}} \frac{\boldsymbol{b} \times \nabla B}{\Omega_{a} B} \frac{Q_{\| a}+Q_{\perp a}}{N_{a} v_{t h \| a}}+v_{t h \| a} \frac{\boldsymbol{b}}{2}\left(1+\frac{\nabla_{\perp}^{2} \phi}{\Omega_{a} B}\right) .
$$

The expression for $C_{a b}^{10}$ is given in appendix $\mathrm{D}$, as well as all the $C_{a b}^{p j}$ coefficients relevant for the present fluid model. The left-hand side of (6.7) describes the convection of $u_{\| a}$, while the first term in the right-hand side is related to pressure and heat flux gradients, the second term to resistivity (collisional effects), the third term consists of high-order terms kept to ensure phase-space conservation properties, and the last term is the parallel fluid acceleration, namely due to parallel electric fields, mirror force and inertia.

The parallel and perpendicular temperature equations are obtained by setting $(p, j)=$ $(2,0)$ and $(0,1)$ respectively in $(4.9)$. This yields for the parallel temperature

$$
\begin{aligned}
\frac{N_{a}}{\sqrt{2}} \frac{\mathrm{d}_{a}^{0} T_{\| a}}{\mathrm{~d} t}= & \sqrt{2} Q_{\perp a} \frac{\nabla_{\|} B}{B}-\frac{N_{a} \nabla_{\perp}^{2} \phi}{\sqrt{2} \Omega_{a} B} \frac{\mathrm{d}_{0 a} T_{\| a}}{\mathrm{~d} t}-2 \frac{N_{a} T_{\| a}}{v_{t h \| a}} \boldsymbol{u}_{a}^{1} \cdot \nabla u_{\| a} \\
& -\nabla \cdot\left(N_{a} T_{\| a} \boldsymbol{u}_{a}^{2 \|}\right)+N_{a} T_{\| a} \frac{\boldsymbol{E}}{B} \cdot \frac{\boldsymbol{b} \times \nabla B}{B}\left(1+\frac{\nabla_{\perp}^{2} \phi}{\Omega_{a} B}\right) \\
& +\sum_{b} C_{a b}^{20} N T_{\| a}+\frac{2 N_{a} T_{\| a}}{v_{t h \| a}}\|\mathcal{A}\|_{a}^{* 10}
\end{aligned}
$$

where

$$
\boldsymbol{u}_{a}^{2 \|}=\frac{Q_{\|} a}{2 N_{a} T_{\| a}} \frac{\boldsymbol{U}_{p a}^{t h}}{v_{t h \| a}}+\frac{\sqrt{2} T_{\| a}}{m_{a}} \frac{\boldsymbol{b} \times \nabla B}{\Omega_{a} B}+\frac{\boldsymbol{b}}{2} \frac{Q_{\| a}}{N_{a} T_{\| a}}\left(1+\frac{\nabla_{\perp}^{2} \phi}{\Omega_{a} B}\right),
$$

and for the perpendicular temperature

$$
N_{a} \frac{\mathrm{d}_{a}^{0}}{\mathrm{~d} t}\left(\frac{T_{\perp a}}{B}\right)+\frac{N_{a} \nabla_{\perp}^{2} \phi}{\Omega_{a} B} \frac{\mathrm{d}_{0 a}}{\mathrm{~d} t}\left(\frac{T_{\perp a}}{B}\right)=\nabla \cdot\left(\frac{N_{a} T_{\perp a}}{B} \boldsymbol{u}_{a}^{2 \perp}\right)-\frac{N_{a} T_{\perp a}}{B} \sum_{b} C_{a b}^{01},
$$

with

$$
\boldsymbol{u}_{a}^{2 \perp}=-\frac{Q_{\perp a}}{N_{a} T_{\perp a}} \frac{\boldsymbol{U}_{p a}^{t h}}{v_{t h \| a}}-\frac{T_{\perp a}}{m_{a}} \frac{\boldsymbol{b} \times \nabla B}{\Omega_{a} B} .
$$


The equations for the evolution of the parallel $Q_{\| a}$ and perpendicular $Q_{\perp a}$ heat fluxes are obtained by setting $(p, j)=(3,0)$ and $(1,1)$ respectively in $(4.9)$, yielding

$$
\begin{aligned}
\frac{\mathrm{d}_{a}^{0} Q_{\| a}}{\mathrm{~d} t}= & -\frac{\mathrm{d}_{0 a}}{\mathrm{~d} t}\left(Q_{\| a} \frac{\nabla_{\perp}^{2} \phi}{\Omega_{a} B}\right)+N_{a} T_{\| a} \sqrt{3} v_{t h \| a} \sum_{b} C_{a b}^{30} \\
& -Q_{\| a} \nabla \cdot \boldsymbol{u}_{a}^{0}-\frac{Q_{\| a} \nabla_{\perp}^{2} \phi}{\Omega_{a} B} \nabla \cdot \boldsymbol{U}_{0 a}-3 \nabla \cdot\left(\boldsymbol{u}_{k a} Q_{\| a}\right) \\
& -\frac{3}{\sqrt{2}}\left(1+\frac{\nabla_{\perp}^{2} \phi}{\Omega_{a} B}\right) \frac{\boldsymbol{E} \cdot \boldsymbol{b} \times \nabla B}{B^{2}} Q_{\| a}+3 \sqrt{2} N_{a} T_{\| a}\|\mathcal{A}\|_{a}^{* 20} \\
& -3 \sqrt{2} N_{a} T_{\| a} \boldsymbol{u}_{a}^{2 \|} \cdot \nabla u_{\| a}-3 \sqrt{2} N_{a} v_{t h \| a} \boldsymbol{u}_{a}^{1} \cdot \nabla T_{\| a},
\end{aligned}
$$

and

$$
\begin{aligned}
\frac{\mathrm{d}_{a}^{0}}{\mathrm{~d} t}\left(\frac{Q_{\perp} a}{B}\right)= & -\frac{\mathrm{d}_{0 a}}{\mathrm{~d} t}\left(\frac{Q_{\perp a}}{B} \frac{\nabla_{\perp}^{2} \phi}{\Omega_{a} B}\right)-\frac{N_{a} v_{t h \| a}}{\sqrt{2}}\left(\boldsymbol{u}_{a}^{1} \cdot \nabla\right) \frac{T_{\perp a}}{B} \\
& +\frac{N_{a} T_{\perp a}}{B}\left(\boldsymbol{u}_{a}^{2 \perp} \cdot \nabla\right) u_{\| a}-\left(\frac{Q_{\perp a}}{B}\right)\left(\nabla \cdot \boldsymbol{u}_{a}^{0}+\frac{\nabla_{\perp}^{2} \phi}{\Omega B} \nabla \cdot \boldsymbol{U}_{0 a}\right) \\
& -\left(\boldsymbol{U}_{k a}+2 \boldsymbol{U}_{\nabla B}\right) \cdot \nabla\left(\frac{Q_{\perp a}}{B}\right)-\frac{\sum_{b} C_{a b}^{11}}{\sqrt{2}} \frac{v_{t h \| a} N_{a} T_{\perp a}}{B} \\
& +\left(\frac{N_{a} T_{\perp a}^{2}}{m_{a}} \frac{\nabla_{\|} B}{B^{2}}+\frac{Q_{\perp a}}{B} \boldsymbol{E} \cdot \frac{\boldsymbol{b} \times \nabla B}{B^{2}}\right)\left(1+\frac{\nabla_{\perp}^{2} \phi}{\Omega_{a} B}\right) .
\end{aligned}
$$

In (6.13) and (6.14) we neglected the higher-order moments with respect to $N^{30}$ and $N^{11}$, an approximation that we will scrutinize in the next section. Equations (6.2)(6.14) constitute a closed set of six coupled nonlinear partial differential equations for both the hydrodynamical variables $n_{a}, u_{\| a}, T_{\| a}, T_{\perp a}$ and the kinetic variables $Q_{\| a}$ and $Q_{\perp a}$.

With respect to previous delta-F (Brizard 1992; Dorland \& Hammett 1993) and full-F gyrofluid models (Madsen 2013), our fluid model, equations (6.2)-(6.14), while neglecting $k_{\perp} \rho_{i} \sim 1$ effects, includes the velocity contributions from the $B_{\|}^{*}$ denominator in the equations of motion (2.15) and (2.16) and includes the effects of full Coulomb collisions up to order $\epsilon_{\nu} \epsilon$. Also, due to the choice of basis functions with shifted velocity arguments $H_{p}\left(s_{\| a}\right)$ instead of $H_{p}\left(v_{\|} / v_{t h a}\right)$, we obtain a set of equations that can efficiently describe both weak flow $\left(u_{\| a} \ll v_{\text {tha }}\right)$ and strong flow $\left(u_{\| a} \sim v_{\text {tha }}\right)$ regimes.

\subsection{High-collisionality regime}

We now consider the high-collisionality regime, where the characteristic fluctuation frequency of the hydrodynamical variables $\omega$

$$
\omega \sim v_{\text {tha }}\left|\nabla_{\|} \ln N_{a}\right| \sim v_{t h a}\left|\nabla_{\|} \ln T_{\| a}\right| \sim v_{t h a}\left|\nabla_{\|} \ln T_{\perp a}\right| \sim\left|\nabla_{\|} u_{\| a}\right| \sim v_{t h a} / L_{\| a},
$$

is much smaller than the collision frequency $v_{a} \simeq v_{a a}$, that is

$$
\delta_{a} \sim \frac{\omega}{v_{a}} \sim \frac{\lambda_{m f p a}}{L_{\| a}} \ll 1,
$$


where the mean free path $\lambda_{m f p a}$ in (6.16) is defined as

$$
\lambda_{m f p a}=v_{t h a} / v_{a a}
$$

Equation (6.16) describes the so-called linear transport regime (Balescu 1988). In this case, the distribution function can be expanded around a Maxwell-Boltzmann equilibrium, according to the Chapman-Enskog asymptotic closure scheme (Chapman 1962) and, to first order in $\delta_{a}$, we have

$$
\left\langle F_{a}\right\rangle \simeq F_{M a}\left[1+\delta_{a} f_{1 a}\left(v_{\|}, \mu, \boldsymbol{R}, t\right)\right]
$$

According to (6.18), all moments $N_{a}^{p j}$ in the Hermite-Laguerre expansion (3.1) with $(p, j) \neq(0,0)$ are order $\delta_{a}$. Since $Q_{\| a}$ and $Q_{\perp a}$ are determined at first order in $\delta_{a}$ only by the moments $(p, j)=(0,0),(3,0),(1,1)$, the truncation of Sec. $(6.1)$, i.e. neglecting $(p, j) \neq(0,0),(3,0),(1,1)$ is justified. For a more detailed discussion on this topic see Balescu (1988). Moreover, in the linear regime, a relationship between the hydrodynamical and kinetic variables can be obtained along the lines of the semicollisional closure. This allows us to express $Q_{\| a}$ and $Q_{\perp a}$ as a function of $N_{a}, u_{\| a}, T_{\| a}$ and $T_{\perp a}$, therefore reducing the number of equations. We now derive this functional relationship.

We consider (6.13)-(6.14) in the linear regime, and neglect the polarization terms that are proportional to $\nabla_{\perp}^{2} \phi /\left(\Omega_{a} B\right)$. This yields $\sqrt{3 / 2} \sum_{b} C_{a b}^{30} / v_{t h \| a} \simeq R_{\| a}$ and $\sum_{b} C_{a b}^{11} /\left(\sqrt{2} v_{t h \| a}\right) \simeq R_{\perp a}$, with $R_{\| a}$ and $R_{\perp a}$ given by

$$
\begin{gathered}
R_{\| a}=\frac{\nabla_{\|} T_{\| a}}{T_{\| a}}+u_{\| a} \frac{\boldsymbol{b} \times \nabla B}{\Omega_{a} B} \cdot\left(\frac{\nabla u_{\| a}}{u_{\| a}}+\frac{\nabla T_{\| a}}{T_{\| a}}\right) \\
R_{\perp a}=\frac{T_{\perp a}}{T_{\| a}} \frac{\nabla_{\|} B}{B}-\frac{1}{2 \sqrt{2}} \nabla_{\|} \ln \frac{T_{\perp a}}{B}-u_{\| a} \frac{\boldsymbol{b} \times \nabla B}{\Omega_{a} B} \cdot\left(\frac{T_{\perp a}}{T_{\| a}} \frac{\nabla u_{\| a}}{u_{\| a}}+\nabla \ln \frac{T_{\perp a}}{B}\right)
\end{gathered}
$$

since $\mathrm{d}_{a}^{0} / \mathrm{d} t \sim \mathrm{d}_{0 a} / \mathrm{d} t \sim \omega$ and $\left(\mathrm{d}^{0} Q_{\|, \perp} / \mathrm{d} t\right) / Q_{\|, \perp a} \sim \delta_{a}^{2} v_{a}$. We compute the guiding-centre moments of the collision operator $C_{a b}^{30}$ and $C_{a b}^{11}$ by truncating the series for the likespecies collision operator in $(3.40)$ at $(l, k, n, q)=(2,1,2,1)$. The resulting $C_{a b}^{p j}$ coefficients are presented in appendix D.

With the expression of $C_{a b}^{30}$ and $C_{a b}^{11}$, we can solve for $Q_{\| a}$ and $Q_{\perp a}$. In the regime $\left(T_{\| a}-T_{\perp a}\right) / T_{a} \sim \delta$, at lowest order, we obtain for the electron species

$$
\frac{Q_{\| e}}{N_{e} T_{e} v_{\text {the }}}=-0.362 \frac{u_{\| e}-u_{\| i}}{v_{\text {the }}}-10.6 \lambda_{m f p e} \frac{\nabla_{\|} T_{e}}{T_{e}},
$$

and

$$
\frac{Q_{\perp e}}{N_{e} T_{e} v_{\text {the }}}=-0.119 \frac{u_{\| e}-u_{\| i}}{v_{\text {the }}}-3.02 \lambda_{m f p e} \frac{\nabla_{\|} T_{e}}{T_{e}} .
$$

Analogous expressions are obtained for the ion species.

Equations (6.2), (6.7), (6.9) and (6.11), with $Q_{\| a}$ and $Q_{\perp a}$ given by (6.21) and (6.22) are valid in the high-collisionality regime, and can be compared with the drift-reduced Braginskii equations in Zeiler et al. (1997). We first rewrite the continuity equation, equation (6.2), in the form 


$$
\frac{\partial N_{e}}{\partial t}+\nabla \cdot\left[N_{e}\left(\boldsymbol{v}_{E}+u_{\| e} \boldsymbol{b}+\frac{T_{\| e}+T_{\perp e}}{m_{e}} \frac{\boldsymbol{b} \times \nabla B}{\Omega_{e} B}\right)\right]=0,
$$

where we expand the convective derivative $\mathrm{d}^{0} a / \mathrm{d} t$ using (6.3) and (6.4), and neglect polarization terms proportional to the electron mass $m_{e}$. By noting that the diamagnetic drift $v_{d e}$ can be written as

$$
\boldsymbol{v}_{d e}=\frac{1}{e N_{e}} \nabla \times \frac{p_{e} \boldsymbol{b}}{B}-2 \frac{T_{e}}{m_{e}} \frac{\boldsymbol{b} \times \nabla B}{\Omega_{e} B},
$$

and by considering the isotropic regime $T_{\| e} \sim T_{\perp e} \sim T_{e}$, we obtain

$$
\frac{\partial N_{e}}{\partial t}+\nabla \cdot\left[N_{e}\left(\boldsymbol{v}_{E}+u_{\| e} \boldsymbol{b}+\boldsymbol{v}_{d e}\right)\right]=0,
$$

which corresponds to the continuity equation in the drift-reduced Braginskii model in Zeiler et al. (1997). In that model, the polarization equation is obtained by subtracting both electron and ion continuity equations, using Poisson's equation $n_{e} \simeq n_{i}$ with $n_{e}$ and $n_{i}$ the electron and ion particle densities respectively, and neglecting the electron to ion mass ratio. Applying the same procedure to the present fluid model, we obtain

$$
\begin{aligned}
0= & \nabla \cdot\left(\frac{\nabla_{\perp}^{2} \phi N_{i} u_{\| i} \boldsymbol{b}}{\Omega_{i} B}\right)-\nabla \cdot\left[\frac{\boldsymbol{v}_{E}}{2 m_{i}} \nabla_{\perp}^{2}\left(\frac{N_{i} T_{\perp i}}{\Omega_{i}^{2}}\right)\right]-\frac{1}{2 m_{i}} \frac{\partial}{\partial t} \nabla_{\perp}^{2}\left(\frac{N_{i} T_{\perp i}}{\Omega_{i}^{2}}\right) \\
& +\nabla \cdot\left(\frac{N_{i}}{\Omega_{i}} \boldsymbol{b} \times \frac{\mathrm{d}_{0 i} \boldsymbol{U}_{0 i}}{\mathrm{~d} t}\right)+\nabla \cdot\left[\boldsymbol{b}\left(N_{i} u_{\| i}-N_{e} u_{\| e}\right)\right] \\
& +\nabla \cdot\left[\left(N_{i} T_{\| i}+N_{e} T_{\| e}+N_{i} T_{\perp i}+N_{e} T_{\perp e}\right) \frac{\boldsymbol{b} \times \nabla B}{e B^{2}}\right] .
\end{aligned}
$$

In (6.26), the first three terms, which are not present in the drift-reduced Braginskii model, correspond to the difference between ion guiding-centre density $N_{i}$ and particle density $n_{i}$, proportional to both $\nabla_{\perp}^{2} \phi$ and $\nabla_{\perp}^{2} P_{i}$. The parallel momentum and temperature equations, equation (6.7) and (6.9), with respect to (Zeiler et al. 1997), contain the higher-order term $\mathcal{A}$ that ensures phase-space conservation, mirror force terms proportional to $\left(\nabla_{\|} B\right) / B$ and polarization terms proportional to $\nabla_{\perp}^{2} \phi /\left(\Omega_{a} B\right)$ due to the difference between guiding-centre and particle fluid quantities. This set of fluid equations constitute an improvement over the drift-reduced Braginskii model. With respect to the original Braginskii equations (Braginskii 1965), they include the nonlinear terms that arise when retaining full Coulomb collisions, and the effect of ion-electron collisions.

\section{Conclusion}

In the present work, a full-F drift-kinetic model is developed, suitable to describe the plasma dynamics in the SOL region of tokamak devices at arbitrary collisionality. Taking advantage of the separation between the turbulent and gyromotion scales, a gyroaveraged Lagrangian and its corresponding equations of motion are obtained. This is the starting point to deduce a drift-kinetic Boltzmann equation with full Coulomb collisions for the gyroaveraged distribution function. 
The gyroaveraged distribution function is then expanded into an Hermite-Laguerre basis, and the coefficients of the expansion are related to the lowest-order gyrofluid moments. The fluid moment expansion of the Coulomb operator described in Ji \& Held (2009) is reviewed, and its respective particle moments are written in terms of coefficients of the Hermite-Laguerre expansion, relating both expansions. This allows us to express analytically the moments of the collision operator in terms of guidingcentre moments. A set of equations, indeed a moment hierarchy that describes the evolution of the guiding-centre moments is derived, together with a Poisson's equation accurate up to $\epsilon^{2}$. These are then used to derive a fluid model in the high-collisionality limit.

The drift-kinetic model derived herein can be considered a starting point for the development of a gyrokinetic Boltzmann equation suitable for the SOL region (e.g. Qin et al. 2007; Hahm, Wang \& Madsen 2009). Indeed, using a similar approach, a gyrokinetic moment hierarchy may be derived, allowing for the use of perpendicular wave numbers satisfying $k_{\perp} \rho_{s} \sim 1$. For a recent Hermite-Laguerre formulation of the nonlinear delta-F gyrokinetic equation see Mandell et al. (2017).

\section{Acknowledgements}

This work has been carried out within the framework of the EUROfusion Consortium and has received funding from the Euratom research and training programme 2014-2018 under grant agreement No 633053, and from Portuguese FCT - Fundação para a Ciência e Tecnologia, under grant PD/BD/105979/2014. N.F.L. was partially funded by US Department of Energy Grant no. DE-FG02-91ER54109. The views and opinions expressed herein do not necessarily reflect those of the European Commission.

\section{Appendix A. Basis transformation}

In the present appendix, we derive the expressions for the coefficients $T_{a l k}^{p j}$ appearing in (3.30). These coefficients allows us to express up to order $\epsilon \epsilon_{v}$ the relation between fluid $\boldsymbol{M}_{a}^{l k}$ and guiding-centre $N_{a}^{l k}$ moments via (3.32). As a first step, we define a transformation similar to (3.30) but with isotropic temperatures between both bases

$$
c_{a}^{l} P_{l}\left(\xi_{a}\right) L_{k}^{l+1 / 2}\left(c_{a}^{2}\right)=\sum_{p=0}^{l+2 k} \sum_{j=0}^{k+\lfloor l / 2\rfloor} \bar{T}_{l k a}^{p j} H_{p}\left(\frac{v_{\|}-u_{\| a}}{v_{\text {tha }}}\right) L_{j}\left(\frac{v_{\perp}^{\prime 2}}{v_{\text {tha }}^{2}}\right),
$$

with the inverse transformation

$$
H_{p}\left(\frac{v_{\|}-u_{\| a}}{v_{\text {tha }}}\right) L_{j}\left(\frac{v_{\perp}^{\prime 2}}{v_{\text {tha }}^{2}}\right)=\sum_{l=0}^{p+2 j} \sum_{k=0}^{j+\lfloor p / 2\rfloor}\left(\bar{T}^{-1}\right)_{p j a}^{l k} c_{a}^{l} P_{l}\left(\xi_{a}\right) L_{k}^{l+1 / 2}\left(c_{a}^{2}\right) .
$$

The relation between the coefficients $\left(\bar{T}^{-1}\right)_{p j}^{l k}$ and $\bar{T}_{l k}^{p j}$ is given by

$$
\left(\bar{T}^{-1}\right)_{p j}^{l k}=\frac{\sqrt{\pi} 2^{p} p !(l+1 / 2) k !}{(k+l+1 / 2) !} \bar{T}_{l k}^{p j}
$$

By integrating both sides of (A 1) over the whole velocity space, the expression for $\bar{T}_{l k}^{p j}$ is obtained 


$$
\begin{aligned}
\bar{T}_{l k}^{p j}= & \sum_{q=0}^{\lfloor l / 2\rfloor} \sum_{v=0}^{\lfloor p / 2\rfloor} \sum_{i=0}^{k} \sum_{r=0}^{q} \sum_{s=0}^{\min (j, i)} \sum_{m=0}^{k-i} \frac{(-1)^{q+i+j+v+m}}{2^{((3 l+p) / 2)+m+v-r}} \\
& \times\left(\begin{array}{c}
l \\
q
\end{array}\right)\left(\begin{array}{c}
2(l-q) \\
l
\end{array}\right)\left(\begin{array}{c}
q \\
r
\end{array}\right)\left(\begin{array}{c}
r \\
j-s
\end{array}\right)\left(\begin{array}{c}
r \\
i-s
\end{array}\right)\left(\begin{array}{c}
s+r \\
s
\end{array}\right) r ! \\
& \times \frac{(k-i+l-1 / 2) !(l+p+2(m-r-v)-1) ! !}{(p-2 v) !(k-i-m) !(l+m-1 / 2) ! v ! m !} .
\end{aligned}
$$

We then integrate both sides of (3.30) with weights $H_{l}\left(s_{\| a}\right) L_{j}\left(s_{\perp a}^{2}\right)$, with the argument transformation

$$
H_{p}\left(s_{\| a}\right)=\left(\frac{T_{a}}{T_{\| a}}\right)^{p / 2} \sum_{k=0}^{\lfloor p / 2\rfloor} \frac{p !}{k !(p-2 k) !}\left(1-\frac{T_{\| a}}{T_{a}}\right)^{k} H_{p-2 k}\left(\frac{v_{\|}-u_{\| a}}{v_{\text {tha }}}\right),
$$

and

$$
L_{j}\left(s_{\perp a}^{2}\right)=\sum_{k=0}^{j}\left(\begin{array}{c}
j \\
j-k
\end{array}\right)\left(\frac{T_{a}}{T_{\perp a}}\right)^{k}\left(1-\frac{T_{a}}{T_{\perp a}}\right)^{j-k} L_{k}\left(\frac{v_{\perp}^{\prime 2}}{v_{t h a}^{2}}\right),
$$

to find the relation between the isotropic and anisotropic temperature coefficients

$$
\begin{aligned}
T_{a l k}^{p j}= & \sum_{p p=0}^{l+2 k} \sum_{j j=0}^{k+\lfloor l] 2} \sum_{z=0}^{j j} \sum_{d=0}^{\lfloor p p / 2\rfloor}\left(\begin{array}{c}
j j \\
j j-z
\end{array}\right) \frac{p p ! \delta_{z, j} \delta_{p, p p-2 d}}{d !(p p-2 d) !} \\
& \times\left(\frac{T_{\| a}}{T_{a}}\right)^{p / 2}\left(\frac{T_{\perp a}}{T_{a}}\right)^{z}\left(1-\frac{T_{a}}{T_{\| a}}\right)^{d}\left(1-\frac{T_{\perp a}}{T_{a}}\right)^{j j-z} \bar{T}_{l k}^{p p j}, \\
\left(T_{a}^{-1}\right)_{p j}^{l k}= & \sum_{z=0}^{j} \sum_{d=0}^{\lfloor p / 2\rfloor} \sum_{l l=0}^{p-2 d+2 z} \sum_{k k=0}^{z-d+\lfloor p\rfloor 2}\left(\begin{array}{c}
j \\
j-z
\end{array}\right) \frac{p ! \delta_{l, l l} \delta_{k, k k}}{d !(p-2 d) !} \\
& \times\left(\frac{T_{a}}{T_{\| a}}\right)^{p / 2}\left(\frac{T_{a}}{T_{\perp a}}\right)^{z}\left(1-\frac{T_{\| a}}{T_{a}}\right)^{d}\left(1-\frac{T_{a}}{T_{\perp a}}\right)^{j-z}\left(\overline{T^{-1}}\right)_{p-2 d z}^{l l k k} .
\end{aligned}
$$

\section{Appendix B. Guiding-centre moments of $\mathcal{A}$}

In (2.16), the term $\mathcal{A}$ that ensures phase-space conservation properties for the particle equations of motion is introduced. Here, we present the analytic expressions for its guiding-centre moments $\|\mathcal{A}\|_{a}^{* p j}$ appearing in (4.12). These are given by

$$
\begin{aligned}
\|\mathcal{A}\|_{a}^{* p j}= & \frac{1}{N_{a} \Omega_{a}} \sum_{l, k}\left(A_{1 a} \mathcal{V}_{l k}^{3 p j}+A_{2 a} \mathcal{V}_{l k}^{2 p j}+A_{3 a} \mathcal{V}_{l k}^{1 p j}\right. \\
& \left.+A_{4 a} \mathcal{V}_{l k}^{1 p^{p^{\prime} j^{\prime}}} \mathcal{M}_{p^{\prime} j^{\prime}}^{p j}+A_{5 a} \mathcal{M}_{l k}^{p j}+A_{6 a} \delta_{p l} \delta_{j k}\right) N_{a}^{l k},
\end{aligned}
$$

with the phase-mixing term

$$
\begin{aligned}
\mathcal{V}_{l k}^{3 p j}= & {\left[\sqrt{(p+3)(p+2)(p+1)} \delta_{p+3, l}+3 \sqrt{(p+1)^{3}} \delta_{p+1, l}\right.} \\
& \left.+3 \sqrt{p^{3}} \delta_{p-1, l}+\sqrt{p(p-1)(p-2)} \delta_{p-3, l}\right] \frac{\delta_{j, k}}{\sqrt{8}},
\end{aligned}
$$


and the coefficients $A_{i}$

$$
\begin{gathered}
A_{1 a}=v_{t h \| a}^{3} \boldsymbol{k}_{\perp} \cdot \boldsymbol{\nabla} \times \boldsymbol{b}, \\
A_{2 a}=v_{t h \| a}^{2}\left[\boldsymbol{k}_{\perp} \cdot\left(u_{\| a} \nabla \times \boldsymbol{b}+\nabla \times \boldsymbol{v}_{E}\right)+\nabla \times \boldsymbol{b} \cdot \boldsymbol{A}\right], \\
A_{3 a}=v_{t h \| a}\left(u_{\| a} \boldsymbol{\nabla} \times \boldsymbol{b}+\boldsymbol{\nabla} \times \boldsymbol{v}_{E}\right) \cdot \boldsymbol{A}+v_{t h \| a}^{2} \boldsymbol{\nabla} \times \boldsymbol{b} \cdot \boldsymbol{C}, \\
A_{4 a}=v_{t h \| a} \frac{T_{\perp}}{m_{a} B} \nabla_{\perp} B \cdot \boldsymbol{\nabla} \times \boldsymbol{b}, \\
A_{5 a}=\frac{T_{\perp a}}{m_{a} B} \nabla_{\perp} B \cdot\left(u_{\| a} \boldsymbol{\nabla} \times \boldsymbol{b}+\boldsymbol{\nabla} \times \boldsymbol{v}_{E}\right), \\
A_{6 a}=\left(v_{t h \| a} u_{\| a} \boldsymbol{\nabla} \times \boldsymbol{b}+\boldsymbol{\nabla} \times \boldsymbol{v}_{E}\right) \cdot \boldsymbol{C}
\end{gathered}
$$

with

$$
\begin{gathered}
\boldsymbol{A}=\left[\frac{\partial \boldsymbol{b}}{\partial t}+(\boldsymbol{b} \cdot \boldsymbol{\nabla}) \boldsymbol{v}_{E}+\left(\boldsymbol{v}_{E} \cdot \nabla\right) \boldsymbol{b}+2 u_{\| a} v_{t h \| a} \boldsymbol{k}\right]_{\perp}, \\
\boldsymbol{C}=\frac{1}{v_{t h \| a}}\left[\frac{\partial \boldsymbol{v}_{E}}{\partial t}+\left(\boldsymbol{v}_{E} \cdot \nabla\right) \boldsymbol{v}_{E}+u_{\| a}^{2} \boldsymbol{k}\right]_{\perp} .
\end{gathered}
$$

\section{Appendix C. Poisson's equation with collisional effects}

To include $\epsilon_{\nu}$ effects in Poisson's equation, we retain the $l=1$ Bessel term in (5.6), yielding

$$
\begin{aligned}
\epsilon_{0} \nabla \cdot \boldsymbol{E}= & \sum_{a} q_{a}\left[N_{a}\left(1+\frac{\boldsymbol{b} \cdot \boldsymbol{\nabla} \times \boldsymbol{b}}{\Omega} u_{\| a}+\frac{\boldsymbol{b} \cdot \boldsymbol{\nabla} \times \boldsymbol{v}_{E}}{\Omega}\right)\right. \\
& \left.+\frac{1}{2 m_{a}} \nabla_{\perp}^{2}\left(\frac{P_{\perp a}}{\Omega_{a}^{2}}\right)+2 \pi \int \Gamma_{1}\left[C_{1 a} \mathrm{e}^{\mathrm{i} \alpha}+C_{-1 a} \mathrm{e}^{-\mathrm{i} \alpha}\right] \frac{B_{\|}^{*}}{m_{a}} \mathrm{~d} v_{\|} \mathrm{d} \mu\right] .
\end{aligned}
$$

The collisional terms $C_{ \pm 1 a}=\sum_{b} C_{ \pm 1 a b}$ (for collisions between species $a$ and $b$ ) can be cast in terms of gyrofluid moments $N_{a}^{l k}$. For like-species collisions, we use (3.22) to express the collision operator $C_{a a 0}$ in (3.21) in terms of fluid moments, together with the property (Ji \& Held 2006)

$$
\boldsymbol{P}^{l}(\boldsymbol{v}) \cdot \boldsymbol{T}^{l k}=\boldsymbol{v}^{l} \cdot \boldsymbol{T}^{l k}
$$

which holds for any totally symmetric and traceless tensor $T^{l k}$. This yields the following form for the lowest-order collision operator (3.22)

$$
\begin{aligned}
c_{0}\left(f_{a}^{l k m}, f_{a}^{n q r}\right)= & f_{a M} \mathcal{N}_{a}^{l k} \mathcal{N}_{a}^{n q} \sum_{u=0}^{\min (2, l, n)} v_{* a a u}^{l m, n r}\left(c^{2}\right) \\
& \times \sum_{i=0}^{\min (l, n)-u} d_{i}^{l-u, n-u} \frac{\boldsymbol{c}_{a}^{l+n-2(i+u)}}{c_{a}^{l+n-2(i+u)}} \cdot \overline{\boldsymbol{P}^{l}(\boldsymbol{b}) \cdot{ }^{i+u} \boldsymbol{P}^{n}(\boldsymbol{b})},
\end{aligned}
$$

as $\overline{\boldsymbol{T}}$ means the traceless symmetrization of $\boldsymbol{T}$. The shifted velocity vector $\boldsymbol{c}_{a}=$ $\left(\boldsymbol{v}-\boldsymbol{u}_{a}\right) / v_{\text {tha }}$, under the transformation of (2.3), with the lowest-order fluid velocity 
$\boldsymbol{u}_{a} \simeq u_{\| a} \boldsymbol{b}+\boldsymbol{v}_{E}$, can be written as

$$
\boldsymbol{c}_{a}=c_{\| a} \boldsymbol{b}+\frac{c_{\perp a}}{2}\left(\mathrm{e}^{\mathrm{i} \theta} \boldsymbol{E}_{1}+\mathrm{e}^{-\mathrm{i} \theta} \boldsymbol{E}_{2}\right)
$$

where $\boldsymbol{E}_{1,2}=\boldsymbol{e}_{2} \pm \mathrm{i} \boldsymbol{e}_{1}$. Using the multinomial theorem

$$
\left(\sum_{i=1}^{m} x_{i}\right)^{k}=\sum_{a_{i} \geqslant 0} \frac{k ! \prod_{i=1}^{m} x_{i}^{a_{i}}}{\prod_{i=1}^{m} a_{i} !}
$$

subject to the constraint $\sum_{i=1}^{m} a_{i}=k$, we obtain

$$
\boldsymbol{c}_{a}^{k}=\sum_{a_{1}+a_{2}+a_{3}=k} \frac{k !\left(c_{\| a}\right)^{a_{1}}}{a_{1} ! a_{2} ! a_{3} !}\left(\frac{c_{\perp a}}{2}\right)^{a_{2}+a_{3}} \mathrm{e}^{\mathrm{i} \theta\left(a_{2}-a_{3}\right)} \boldsymbol{b}^{a_{1}} \boldsymbol{E}_{1}^{a_{2}} \boldsymbol{E}_{2}^{a_{3}} .
$$

We use (C6) to make explicit the dependence of $\boldsymbol{c}_{a}$ on $\theta$ in (C 3). The Fourier components $C_{1 a}$ and $C_{-1 a}$ correspond to the case $a_{2}=a_{3} \pm 1$ in the sum in (C 6) above, yielding

$$
\begin{aligned}
C_{ \pm 1 a a}= & \sum_{l, k, m} \sum_{n, q, r} \frac{L_{k m}^{l} L_{q r}^{n}}{\sqrt{\sigma_{k}^{l} \sigma_{q}^{n}}} f_{a M} \mathcal{N}_{a}^{l k} \mathcal{N}_{a}^{n q} \sum_{u=0}^{\min (2, l, n)} v_{* a a u}^{l m, n r}\left(c_{a}^{2}\right) \sum_{i=0}^{\min (l, n)-u} d_{i}^{l-u, n-u} \\
& \times \sum_{\substack{a_{1}+a_{2}+a_{3}=\\
l+n-2(i+u)}} \frac{(l+n-2(i+u)) !}{a_{1} ! a_{2} ! a_{3} !}\left(c_{\| a}\right)^{a_{1}}\left(\frac{c_{\perp a}}{2}\right)^{a_{2}+a_{3}} \delta_{a_{2}, a_{3} \pm 1} \\
& \times \frac{\boldsymbol{b}^{a_{1}} \boldsymbol{E}_{1}^{a_{2}} \boldsymbol{E}_{2}^{a_{3}}}{\left(c_{a}\right)^{l+n-2(i+u)}} \cdot \overline{\boldsymbol{P}^{l}(\boldsymbol{b}) \cdot{ }^{i+u} \boldsymbol{P}^{n}(\boldsymbol{b})} .
\end{aligned}
$$

Assembling the velocity dependent terms of $(\mathrm{C} 7)$, together with $J_{1}\left(k_{\perp} \rho_{a}\right) \simeq$ $k_{\perp} v_{\text {tha }} c_{\perp a} /\left(2 \Omega_{a}\right)$, the velocity integration of the like-species operator in Poisson's equation $(\mathrm{C} 1)$ is then

$$
\begin{aligned}
I_{a a}^{ \pm}= & \int c_{\perp a} C_{ \pm 1 a a} \frac{B_{\|}^{*}}{m_{a}} \mathrm{~d} v_{\|} \mathrm{d} \mu=\sum_{l k m} \sum_{n q r} \sum_{u=0}^{\min (2, l, n)} \sum_{i=0}^{\min (l, n)-u} \sum_{a_{1}+a_{2}+a_{3}=l+n-2(i+u)} \\
& \times \frac{L_{k m}^{l} L_{q r}^{n}}{\sqrt{\sigma_{k}^{l} \sigma_{q}^{n}}} \mathcal{N}_{a}^{l k} \mathcal{N}_{a}^{n q} d_{i}^{l-u, n-u} \delta_{a_{2}, a_{3} \pm 1} \frac{(l+n-2(i+u)) !}{a_{1} ! a_{2} ! a_{3} ! 2^{a_{2}+a_{3}}} \\
& \times \boldsymbol{b}^{a_{1}} \boldsymbol{E}_{1}^{a_{2}} \boldsymbol{E}_{2}^{a_{3}} \cdot \overline{\boldsymbol{P}^{l}(\boldsymbol{b}) \cdot{ }^{i+u} \boldsymbol{P}^{n}(\boldsymbol{b})} v_{t h a}^{3} I_{a \pm},
\end{aligned}
$$

where

$$
I_{a \pm}=\int f_{M a} c_{\perp a}\left(c_{\| a}\right)^{a_{1}}\left(c_{\perp a}\right)^{2 a_{2} \pm 1} \frac{\nu_{* a a u}^{l m, n r}\left(c_{a}^{2}\right)}{c_{a}^{l+n-2(i+u)}} \frac{B_{\|}^{*}}{m_{a}} \mathrm{~d} v_{\|} \mathrm{d} \mu .
$$

Converting to pitch-angle coordinates $v_{\|}=v_{\text {tha }} c_{\| a}=v_{\text {tha }} \xi_{a} c_{a}, v_{\perp}^{\prime 2}=v_{\text {tha }}^{2} c_{\perp a}^{2}=c_{a}^{2}\left(1-\xi_{a}^{2}\right)$, with the volume element $\left(B_{\|}^{*} / m_{a}\right) \mathrm{d} \mu \mathrm{d} v_{\|}=v_{\text {tha }}^{3} c_{a}^{2} \mathrm{~d} c_{a} \mathrm{~d} \xi_{a}$, the integral of (C 9) can be 
performed analytically, yielding

$$
\begin{aligned}
& I_{a \pm}=\int_{-1}^{1} \xi_{a}^{a_{1}}\left(1-\xi_{a}^{2}\right)^{a_{3}+\sigma_{ \pm}} \mathrm{d} \xi_{a} \int f_{M a} v_{* a a u}^{l m, n r} c^{2\left(a_{3}+\sigma_{ \pm}+i+u\right)+a_{1}-l-n} \frac{\mathrm{d}^{3} \boldsymbol{c}}{4 \pi}
\end{aligned}
$$

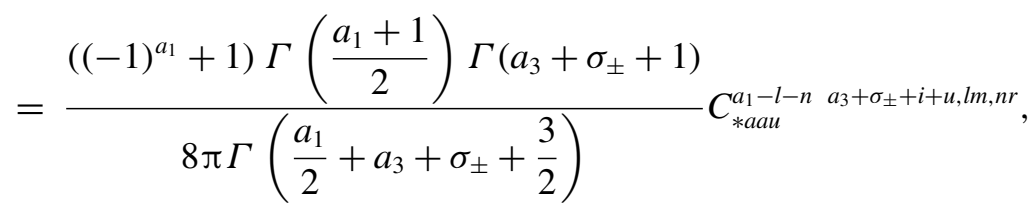

where $\sigma_{ \pm}=(1 \pm 1) / 2$ for $C_{ \pm 1 a a}$ respectively.

For electron-ion collisions, we take the expression for $C_{e i}=C_{e i}^{0}+C_{e i}^{1}$ given by equations (2.25) and (2.26) respectively. By using the shifted velocity vector $\boldsymbol{c}_{a}$ of (C4), we can proceed as for the like-species operator, yielding the Fourier components $C_{ \pm 1 e i}=C_{ \pm 1 e i}^{0}+C_{ \pm 1 e i}^{1}$, with

$$
\begin{aligned}
C_{ \pm 1 e i}^{0}= & -\sum_{l, k} \frac{n_{i} L_{e i}}{8 \pi c_{e}^{3}} \frac{l(l+1) f_{e M} \mathcal{N}_{e}^{l k}}{\sqrt{\sigma_{k}^{l}}} \\
& \times \sum_{a_{1}+a_{2}+a_{3}=l} \frac{l !\left(c_{\| e}\right)^{a_{1}}}{a_{1} ! a_{2} ! a_{3} !}\left(\frac{c_{\perp e}}{2}\right)^{a_{2}+a_{3}} \boldsymbol{b}^{a_{1}} \boldsymbol{E}_{1}^{a_{2}} \boldsymbol{E}_{2}^{a_{3}} \cdot \boldsymbol{P}^{l}(\boldsymbol{b}) \delta_{a_{2}, a_{3} \pm 1},
\end{aligned}
$$

and

$$
C_{ \pm 1 e i}^{1}=f_{M e} \frac{n_{i} L_{e i} m_{e}}{8 \pi c_{e}^{3} T_{e}} \frac{c_{\perp e}}{2} \boldsymbol{u}_{e i} \cdot \boldsymbol{E}_{1,2} .
$$

The velocity integration of $C_{ \pm 1 e i}^{0}$ and $C_{ \pm 1 e i}^{1}$ are given by

$$
\begin{aligned}
I_{e i}^{0 \pm}= & \int c_{\perp e} C_{ \pm 1 e i}^{0} \frac{B_{\|}^{*}}{m_{a}} \mathrm{~d} v_{\|} \mathrm{d} \mu \\
= & -\sum_{l, k} \sum_{a_{1}+a_{2}+a_{3}=l} \delta_{a_{2}, a_{3} \pm 1} \frac{v_{e i} n_{e} v_{t h e}^{3} \mathcal{N}_{e}^{l k}}{16 \pi^{3 / 2} \sqrt{\sigma_{k}^{l}} 2^{a_{2}+a_{3}}} \frac{l(l+1) l !}{a_{1} ! a_{2} ! a_{3} !} \\
& \times \frac{\Gamma\left(\frac{a_{1}+a_{3}+\sigma_{ \pm}}{2}\right) \Gamma\left(\frac{a_{1}+1}{2}\right) \Gamma\left(a_{3}+\sigma_{ \pm}+1\right)}{\Gamma\left(\frac{3}{2}+\frac{a_{1}}{2}+a_{3}+\sigma_{ \pm}\right)}
\end{aligned}
$$

and

$$
I_{e i}^{1 \pm}=\int c_{\perp e} C_{ \pm 1 e i}^{1} \frac{B_{\|}^{*}}{m_{a}} \mathrm{~d} v_{\|} \mathrm{d} \mu=\frac{n_{e} v_{e i}}{6 \sqrt{\pi}} v_{t h e}^{2} \boldsymbol{u}_{e i} \cdot \boldsymbol{E}_{1,2},
$$

respectively. Ion-electron collisions are neglected due to the smallness of the electron to ion mass ratio. Poisson's equation including $\epsilon^{2}$ and $\epsilon_{\nu} \epsilon$ effects then reads

$$
\begin{aligned}
\epsilon_{0} \boldsymbol{\nabla} \cdot \boldsymbol{E}= & \sum_{a} q_{a}\left[N_{a}\left(1+\frac{\boldsymbol{b} \cdot \boldsymbol{\nabla} \times \boldsymbol{b}}{\Omega_{a}} u_{\| a}+\frac{\boldsymbol{b} \cdot \boldsymbol{\nabla} \times \boldsymbol{v}_{E}}{\Omega_{a}}\right)\right. \\
& \left.+\frac{1}{2 m_{a}} \nabla_{\perp}^{2}\left(\frac{P_{\perp a}}{\Omega_{a}^{2}}\right)+\sum_{b} \frac{\pi v_{t h a}}{\Omega_{a}} \int k_{\perp}\left(\mathrm{e}^{\mathrm{i} \alpha} I_{a b}^{+}+\mathrm{e}^{-\mathrm{i} \alpha} I_{a b}^{-}\right) \mathrm{e}^{\mathrm{i} k \cdot \boldsymbol{x}} \mathrm{d}^{3} \boldsymbol{k}\right] .
\end{aligned}
$$




\section{Appendix D. Expressions for the moments of the collision operator}

In the present appendix, we present the expressions for the guiding-centre moments of the collision operator relevant for the fluid model in $\S 6$. The collision operator moments satisfy particle conservation

$$
C_{a b}^{00}=0
$$

and momentum conservation at lowest order

$$
\begin{gathered}
C_{a a}^{10}=0, \\
C_{e i}^{10}=-\frac{m_{i}}{m_{e}} \frac{v_{t h \| i}}{v_{t h \| e}} C_{i e}^{10}+O\left(m_{e} / m_{i}\right) .
\end{gathered}
$$

Both the like species and electron-ion collisions satisfy energy conservation exactly, while the ion-electron operator satisfies (D4) at zeroth order in $\delta_{a}$

$$
T_{\| a} C_{a b}^{20}-\sqrt{2} T_{\perp a} C_{a b}^{01}=0
$$

The remaining moments $C_{a b}^{p j}$, in the linear transport regime with $\Delta T_{a} / T_{a}=\left(T_{\| a}-\right.$ $\left.T_{\perp a}\right) T_{a} \sim N^{11} \sim N^{30} \sim\left(u_{\| e}-u_{\| i}\right) / v_{\text {the }} \sim \delta_{a}$, for ion-electron collisions are given by

$$
\begin{gathered}
C_{i e}^{10}=-\frac{m_{e}}{m_{i}} \frac{v_{t h \| i}}{v_{t h \| e}} C_{e i}^{10}, \\
C_{i e}^{20}=\sqrt{2} v_{e i} \frac{m_{e}}{m_{i}}\left(\frac{T_{e}-T_{i}}{T_{i}}\right)-\frac{2 \sqrt{2} v_{e i}}{3} \frac{m_{e}}{m_{i}} \frac{T_{e}}{T_{i}} \frac{\Delta T_{i}}{T_{i}}, \\
C_{i e}^{01}=-2 v_{e i} \frac{m_{e}}{m_{i}}\left(\frac{T_{e}-T_{i}}{T_{i}}\right)-\frac{2 v_{e i}}{3} \frac{m_{e}}{m_{i}} \frac{T_{e}}{T_{i}} \frac{\Delta T_{i}}{T_{i}}, \\
C_{i e}^{30}=-v_{e i} \sqrt{\frac{3}{2}} \frac{m_{e}}{m_{i}} \frac{Q_{\| i}}{n T_{i} v_{t h i}}, \\
C_{i e}^{11}=3 v_{e i} \frac{m_{e}}{m_{i}} \frac{Q_{\perp i}}{n T_{i} v_{t h i}},
\end{gathered}
$$

for electron-ion collisions

$$
\begin{gathered}
C_{e i}^{10}=-\frac{\sqrt{2} v_{e i}}{6 \pi^{3 / 2}} \frac{u_{\| e}-u_{\| i}}{v_{t h e}}+\frac{\sqrt{2} v_{e i}}{10 \pi^{3 / 2}} \frac{Q_{\| e}+2 Q_{\perp e}}{n T_{e} v_{t h e}}, \\
C_{e i}^{20}=-\frac{2 \sqrt{2} v_{e i}}{15 \pi^{3 / 2}} \frac{\Delta T_{e}}{T_{e}} \\
C_{e i}^{30}=\frac{\sqrt{3} v_{e i}}{10 \pi^{3 / 2}} \frac{u_{\| e}-u_{\| i}}{v_{t h e}}-\frac{v_{e i}}{70 \sqrt{3} \pi^{3 / 2}} \frac{31 Q_{\| e}-2 Q_{\perp e}}{n T_{e} v_{t h e}}, \\
C_{e i}^{11}=\frac{v_{e i}}{5 \sqrt{2} \pi^{3 / 2}} \frac{u_{\| e}-u_{\| i}}{v_{t h e}}+\frac{v_{e i}}{150 \sqrt{2} \pi^{3 / 2}} \frac{Q_{\| e}-94 Q_{\perp e}}{n T_{e} v_{t h e}},
\end{gathered}
$$

and for like-species collisions

$$
C_{a a}^{20}=0
$$




$$
\begin{aligned}
C_{a a}^{30} & =-\frac{2 \sqrt{2}}{125 \sqrt{3} \pi^{3 / 2}} \frac{v_{a a}}{n T_{a} v_{t h a}}\left(19 Q_{\| a}-7 Q_{\perp a}\right), \\
C_{a a}^{11} & =-\frac{2}{375 \pi^{3 / 2}} \frac{v_{a a}}{n T_{a} v_{t h a}}\left(7 Q_{\| a}-121 Q_{\perp a}\right) .
\end{aligned}
$$

\section{REFERENCES}

Abel, I. G., Plunk, G. G., Wang, E., Barnes, M., Cowley, S. C., Dorland, W. \& Schekochinin, A. A. 2013 Multiscale gyrokinetics for rotating tokamak plasmas: fluctuations, transport and energy flows. Rep. Prog. Phys. 76 (11), 116201.

Abramowicz, M., Czerny, B., Lasota, J. \& Szuszkiewicz, E. 1988 Slim accretion disks. Astrophys. J. 332, 646.

Abramowitz, M., Stegun, I. \& Miller, D. 1965 Handbook of Mathematical Functions With Formulas, Graphs and Mathematical Tables, National Bureau of Standards Applied Mathematics Series No. 55, vol. 32. United States Department of Commerce, National Institute of Standards and Technology (NBS).

Agostini, M., Terry, J., Scarin, P. \& Zweben, S. 2011 Edge turbulence in different density regimes in Alcator C-Mod experiment. Nucl. Fusion 51 (5), 053020.

Armstrong, T. 1967 Numerical Studies of the Nonlinear Vlasov Equation. Phys. Fluids 10 (6), 1269.

BALesCU, R. 1988 Transport Processes in Plasmas. North-Holland.

Batishchev, O. V., Krasheninnikov, S. I., Catto, P. J., Batishcheva, A. A., Sigmar, D. J., Xu, X. Q., Byers, J. A., Rognlien, T. D., Cohen, R. H., Shoucri, M. M. et al. 1997 Kinetic effects in tokamak scrape-off layer plasmas. Phys. Plasmas 4 (5/2), 1672-1680.

Battaglia, D. J., Burrell, K. H., Chang, C. S., Ku, S., Degrassie, J. S. \& Grierson, B. A. 2014 Kinetic neoclassical transport in the H-mode pedestal. Phys. Plasmas 21 (7), 072508.

Beer, M. \& Hammett, G. 1996 Toroidal gyrofluid equations for simulations of tokamak turbulence. Phys. Plasmas 3 (1996), 4046.

BraginskiI, S. I. 1965 Transport Processes in a Plasma. Consultants Bureau.

BRIZARD, A. 1992 Nonlinear gyrofluid description of turbulent magnetized plasmas. Phys. Fluids B 4 (1992), 1213.

Camporeale, E., Delzanno, G. L., Bergen, B. K. \& Moulton, J. D. 2016 On the velocity space discretization for the Vlasov-Poisson system: comparison between implicit Hermite spectral and Particle-in-Cell methods. Comput. Phys. Commun. 198, 47-58.

Carralero, D., Birkenmeier, G., Müller, H., Manz, P., Demarne, P., Müller, S., Reimold, F., Stroth, U., Wischmeier, M. \& Wolfrum, E. 2014 An experimental investigation of the high density transition of the scrape-off layer transport in ASDEX Upgrade. Nucl. Fusion 54 (12), 123005.

CArreras, B. 2005 Plasma edge cross-field transport: experiment and theory. J. Nucl. Mater. 337-339 (1-3), 315-321.

CARY, J. \& Brizard, A. 2009 Hamiltonian theory of guiding-center motion. Rev. Mod. Phys. 81 (2), 693-738.

Catto, P. J. \& Simakov, A. N. 2004 A drift ordered short mean free path description for magnetized plasma allowing strong spatial anisotropy. Phys. Plasmas 11 (1), 90-102.

Chang, C., Ku, S., Tynan, G., Hager, R., Churchill, R., Cziegler, I., Greenwald, M., Hubbard, A. \& Hughes, J. 2017 Fast low-to-high confinement mode bifurcation dynamics in a tokamak edge plasma gyrokinetic simulation. Phys. Rev. Lett. 118 (17), 175001.

Chapman, S. 1962 The Mathematical Theory of Non-Uniform Gases.

D'Ippolito, D., MYra, J. \& KRAsheninnikov, S. 2002 Cross-field blob transport in tokamak scrape-off-layer plasmas. Phys. Plasmas 9 (1), 222.

D'Ippolito, D., MYra, J. \& ZWeben, S. 2011 Convective transport by intermittent blob-filaments: comparison of theory and experiment. Phys. Plasmas 18 (6), 060501. 
Dorland, W. \& Hammett, G. W. 1993 Gyrofluid turbulence models with kinetic effects. Phys. Fluids B-Plasma Phys. 5 (1993), 812-835.

Dubin, D., Krommes, J. \& Oberman, C. 1983 Nonlinear gyrokinetic equations. Phys. Fluids 26 (12), 3524.

Dudson, B., Umansky, M., Xu, X., Snyder, P. \& Wilson, H. 2009 BOUT++: a framework for parallel plasma fluid simulations. Comput. Phys. Commun. 180 (9), 1467-1480.

Easy, L., Militello, F., Omotani, J., Dudson, B., HavlíčKová, E., Tamain, P., Naulin, V. \& NiELSEN, A. H. 2014 Three dimensional simulations of plasma filaments in the scrape off layer: a comparison with models of reduced dimensionality. Phys. Plasmas 21 (12), 122515.

Endler, M., Niedermeyer, H., Giannone, L., Kolzhauer, E., Rudyj, A., Theimer, G. \& TsoIs, N. 1995 Measurements and modelling of electrostatic fluctuations in the scrape-off layer of ASDEX. Nucl. Fusion 35 (11), 1307-1339.

Erents, S., Chankin, A., Matthews, G. \& Stangeby, P. 2000 Parallel flow in the JeT scrape-off layer. Plasma Phys. Control. Fusion 42 (8), 905-915.

Garcia, O., HoraceK, J. \& PitTs, R. A. 2015 Intermittent fluctuations in the TCV scrape-off layer. Nucl. Fusion 55 (6), 062002.

Grad, H. 1949 On the kinetic theory of rarefied gases. Commun. Pure Appl. Math. 2 (4), 331-407.

GRANT, F. C. \& FEIX, M. C. 1967 Fourier-Hermite solutions of the Vlasov equations in the linearized limit. Phys. Fluids 10 (4), 696-702.

Grošelu, D., Cerri, S. S., Navarro, A. B., Willmott, C., Told, D., Loureiro, N. F., Califano, F. \& Jenko, F 2017 Fully kinetic versus reduced-kinetic modeling of collisionless plasma turbulence. Astrophys. J. 847 (1), 28.

Grulke, O., Terry, J. L., Cziegler, I., Labombard, B. \& Garcia, O. E. 2014 Experimental investigation of the parallel structure of fluctuations in the scrape-off layer of Alcator C-Mod. Nucl. Fusion 54 (4), 43012.

HАнм, T. 1988 Nonlinear gyrokinetic equations for tokamak microturbulence. Phys. Fluids 31 (9), 2670-2673.

HAhM, T. S., WANG, L. \& MADSEN, J. 2009 Fully electromagnetic nonlinear gyrokinetic equations for tokamak edge turbulence. Phys. Plasmas 16 (2), 022305.

Halpern, F., Ricci, P., Jolliet, S., Loizu, J., Morales, J., Mosetto, A., Musil, F., Riva, F., Tran, T. \& Wersal, C. 2016 The GBS code for tokamak scrape-off layer simulations. J. Comput. Phys. 315, 388-408.

Hammett, G., Dorland, W. \& Perkins, F. 1992 Fluid models of phase mixing, Landau damping, and nonlinear gyrokinetic dynamics. Phys. Fluids B 4 (7), 2052-2061.

Hammett, G. W., Beer, M. A., Dorland, W. D., Cowley, S. C. \& Smith, S. A. 1993 Developments in the gyrofluid approach to Tokamak turbulence simulations. Plasma Phys. Control. Fusion 35 (8), 973-985.

Hatch, D. R., Jenko, F., Navarro, A. B., Bratanov, V., Terry, P. W. \& Pueschel, M. J. 2016 Linear signatures in nonlinear gyrokinetics: interpreting turbulence with pseudospectra. New J. Phys. 18 (7), 075018.

Hazeltine, R. D. 1998 Transport theory in the collisionless limit. Phys. Plasmas 5 (9), 3282.

Hazeltine, R. D. \& Meiss, J. D. 2003 Plasma Confinement. Dover.

Helander, P. \& Sigmar, D. 2005 Collisional Transport in Magnetized Plasmas, Cambridge Monographs on Plasma Physics. Cambridge University Press.

Held, M., Wiesenberger, M., Madsen, J. \& Kendl, A. 2016 The influence of temperature dynamics and dynamic finite ion Larmor radius effects on seeded high amplitude plasma blobs. Nucl. Fusion 56 (12), 126005.

Hidalgo, C., Gonçalves, B., Pedrosa, M., Castellano, J., Erents, K., Fraguas, L., Hron, M., Jimenez, J., Matthews, G., VAn Milligen, B. et al. 2002 Empirical similarity in the probability density function of turbulent transport in the edge plasma region in fusion plasmas. Plasma Phys. Control. Fusion 44, 1557-1564.

Hinton, F. \& Hazeltine, R. D. 1976 Theory of plasma transport in toroidal confinements systems. Rev. Mod. Phys. 48 (2), 239-308. 
Hirvijoki, E., Lingam, M., Pfefferlé, D., Comisso, L., Candy, J. \& Bhattacharjee, A. 2016 Fluid moments of the nonlinear Landau collision operator. Phys. Plasmas 23 (8), 1-10.

JACKson, J. 1998 Classical electrodynamics, 3rd Edition. Am. J. Phys. 841 (1999), 159.

JI, J.-Y. \& Held, E. D. 2006 Exact linearized Coulomb collision operator in the moment expansion. Phys. Plasmas 13 (10), 102103.

Ji, J.-Y. \& Held, E. D. 2008 Landau collision operators and general moment equations for an electron-ion plasma. Phys. Plasmas 15 (10), 102101.

Ji, J.-Y. \& Held, E. D. 2009 Full Coulomb collision operator in the moment expansion. Phys. Plasmas 16 (10), 102108.

JI, J.-Y. \& HELD, E. D. 2010 Analytical solution of the kinetic equation for a uniform plasma in a magnetic field. Phys. Rev. E 82 (1), 1-5.

Jorge, R., Ricci, P., Halpern, F., Loureiro, N. F. \& Silva, C. 2016 Plasma turbulence in the scrape-off layer of the ISTTOK tokamak. Phys. Plasmas 23 (10), 102511.

Kočan, M., Gunn, J., Carpentier-Chouchana, S., Herrmann, A., Kirk, A., Komm, M., MÜller, H., Pascal, J., Pitts, R., Rohde, V. et al. 2011 Measurements of ion energies in the tokamak plasma boundary. J. Nucl. Mater. 415 (1 suppl), S1133-S1138.

Krommes, J. A. 2013 The physics of the second-order gyrokinetic magnetohydrodynamic Hamiltonian: $\mu$ conservation, Galilean invariance, and ponderomotive potential. Phys. Plasmas 20 (12), 124501.

LaBombard, B., Boivin, R., Greenwald, M., Hughes, J., Lipschultz, B., Mossessian, D., Pitcher, C., Terry, J. \& Zweben, S. 2001 Particle transport in the scrape-off layer and its relationship to discharge density limit in Alcator C-Mod. Phys. Plasmas 8 (5 II), 2107-2117.

Labombard, B., Hughes, J., Mossessian, D., Greenwald, M., Lipschultz, B., Terry, J. \& THE Alcator C-Mod TEAm 2005 Evidence for electromagnetic fluid drift turbulence controlling the edge plasma state in the Alcator C-Mod tokamak. Nucl. Fusion 45 (12), 1658.

LeE, W. 1983 Gyrokinetic approach in particle simulation. Phys. Fluids 26 (2), 556.

Leonard, A. W. 2014 Edge-localized-modes in tokamaks. Phys. Plasmas 21 (9), 090501.

Liang, Y., Koslowski, H. R., Thomas, P. R., Nardon, E., Alper, B., Andrew, P., Andrew, Y., Arnoux, G., BARAnov, Y., BÉCoulet, M. et al. 2007 Active control of type-I edgelocalized modes with $\mathrm{n}=1$ perturbation fields in the JET tokamak. Phys. Rev. Lett. 98 (26), 265004.

Littlejohn, R. G. 1983 Variational principles of guiding centre motion. J. Plasma Phys. 29 (1), $111-125$.

Lönnroth, J. S., Bateman, G., Bécoulet, M., Beyer, P., Corrigan, G., Figarella, C., Fundamenski, W., Garcia, O. E., Garbet, X., Huysmans, G. et al. 2006 Integrated ELM modelling. Contrib. Plasma Phys. 46 (7-9), 726-738.

Loureiro, N. F., Dorland, W., Fazendeiro, L., Kanekar, A., Mallet, A., Vilelas, M. S. \& ZocCO, A. 2016 Viriato: A Fourier-Hermite spectral code for strongly magnetized fluid-kinetic plasma dynamics. Comput. Phys. Commun. 206, 45-63.

Loureiro, N. F., ScheKochinin, A. A.\& ZocCo, A. 2013 Fast collisionless reconnection and electron heating in strongly magnetized plasmas. Phys. Rev. Lett. 111 (2), 025002.

Madsen, J. 2013 Full-F gyrofluid model. Phys. Plasmas 20 (7), 072301.

Madsen, J., Naulin, V., Nielsen, A. H. \& Rasmussen, J. J. 2016 Collisional transport across the magnetic field in drift-fluid models. Phys. Plasmas 23 (3), 032306.

Mandell, N. R., Dorland, W. \& Landreman, M. 2017 Laguerre-Hermite pseudo-spectral velocity formulation of gyrokinetics. arXiv:1708.04029.

Martin, Y., TAKizuKa, T. \& Group, the ITPA CDBM H-Mode Threshold Data 2008 Power requirement for accessing the H-mode in ITER. J. Phys.: Conf. Ser. 123, 012033.

MikhailovskiI, A. \& TSYPIN, V. 1971 Transport equations and gradient instabilities in a high pressure collisional plasma. Plasma Phys. 13 (9), 785-798.

Mosetto, A., Halpern, F., Jolliet, S., Loizu, J. \& Ricci, P. 2015 Finite ion temperature effects on scrape-off layer turbulence. Phys. Plasmas 22 (1), 0-17.

Myra, J., Davis, W., D’Ippolito, D., Labombard, B., Russell, D., Terry, J. \& Zweben, S. 2013 Edge sheared flows and the dynamics of blob-filaments. Nucl. Fusion 53 (7), 073013. 
Nespoli, F., Furno, I., Labit, B., Ricci, P., Avino, F., Halpern, F., Musil, F. \& Riva, F. 2017 Blob properties in full-turbulence simulations of the TCV scrape-off layer. Plasma Phys. Control. Fusion 59 (5), 055009.

NG, C. S., Bhattacharjee, A. \& Skiff, F. 1999 Kinetic eigenmodes and discrete spectrum of plasma oscillations in a weakly collisional plasma. Phys. Rev. Lett. 83 (10), 1974-1977.

Omotani, J., Dudson, B., HavlíčKovÁ, E. \& Umansky, M. 2015 Non-local parallel transport in BOUT++. J. Nucl. Mater. 463, 769-772.

PARKER, J. T. 2016 Gyrokinetic simulations of fusion plasmas using a spectral velocity space representation. arXiv: 1603.04727.

PARKer, J. T. \& Dellar, P. J. 2015 Fourier-Hermite spectral representation for the Vlasov-Poisson system in the weakly collisional limit. J. Plasma Phys. 2 (2011), 1-36.

PAŠKAUSKAS, R. \& DE NINNO, G. 2009 Lyapunov stability of Vlasov equilibria using FourierHermite modes. Phys. Rev. E 80 (3), 036402.

Pitts, R., Alberti, S., Blanchard, P., Horacek, J., Reimerdes, H. \& Stangeby, P. 2003 ELM driven divertor target currents on TCV. Nucl. Fusion 43 (10), 1145-1166.

QIn, H., Cohen, R., Nevins, W. \& XU, X. 2007 Geometric gyrokinetic theory for edge plasmas. Phys. Plasmas 14 (5), 056110.

Ribeiro, T. \& SCOTT, B. 2008 Gyrofluid turbulence studies of the effect of the poloidal position of an axisymmetric Debye sheath. Plasma Phys. Control. Fusion 50 (5), 055007.

Ritz, C., Brower, D., Rhodes, T., Bengtson, R., Levinson, S., Luhmann, N., Peebles, W. \& Powers, E. 1987 Characterization of tokamak edge turbulence by far-infrared laser scattering and Langmuir probes. Nucl. Fusion 27 (7), 1125-1134.

Rossel, J., Moret, J., Coda, S., Sauter, O., Goodman, T., Felici, F., Testa, D. \& Martin, Y. 2012 Edge-localized mode control by electron cyclotron waves in a tokamak plasma. Nucl. Fusion 52 (3), 2-6.

Schekochihin, A. A., Parker, J. T., Highcock, E. G., Dellar, P. J., Dorland, W. \& HAMmetT, G. 2016 Phase mixing versus nonlinear advection in drift-kinetic plasma turbulence. J. Plasma Phys. 82 (02), 905820212.

Serianni, G., Agostini, M., Antoni, V., Cavazzana, R., Martines, E., Sattin, F., Scarin, P., Spada, E., Spolaore, M., Vianello, N. et al. 2007 Coherent structures and transport properties in magnetized plasmas. Plasma Phys. Control. Fusion 49 (12B), B267-B280.

Shi, E., HAKim, A. \& Hammett, G. 2015 A gyrokinetic one-dimensional scrape-off layer model of an edge-localized mode heat pulse. Phys. Plasmas 22 (2), 022504.

Shi, E. L., Hammett, G. W., Stoltzfus-Dueck, T. \& Hakim, A. 2017 Gyrokinetic continuum simulation of turbulence in a straight open-field-line plasma. J. Plasma Phys. 83 (03), 905830304.

Silva, C., Figueiredo, H., Duarte, P. \& Fernandes, H. 2011 Poloidal asymmetries in the ISTTOK edge plasma. J. Nucl. Mater. 415 (1), S455-S458.

SNYDER, P. \& HAMmetT, G. 2001 A Landau fluid model for electromagnetic plasma microturbulence. Phys. Plasmas 8 (7), 3199-3216.

Stangeby, P. 2000 The Plasma Boundary of Magnetic Fusion Devices, Series in Plasma Physics, vol. 43. CRC Press.

Sugama, H., Watanabe, T.-H. \& Horton, W. 2001 Collisionless kinetic-fluid closure and its application to the three-mode ion temperature gradient driven system. Phys. Plasmas 8 (6), 2617-2628.

Tamain, P., Ghendrih, P., Tsitrone, E., Sarazin, Y., Garbet, X., Grandgirard, V., Gunn, J., Serre, E., Ciraolo, G. \& Chiavassa, G. 2009 3D modelling of edge parallel flow asymmetries. J. Nucl. Mater. 390-391 (1), 347-350.

TASSI, E. 2016 Hamiltonian reduced fluid model for plasmas with temperature and heat flux anisotropies. Theor. Math. Phys. 188 (3), 1377-1393.

Terry, J. L., Zweben, S. J., Umansky, M. V., CZiegler, I., Grulke, O., Labombard, B. \& STOTLER, D. P. 2009 Spatial structure of scrape-off-layer filaments near the midplane and X-point regions of Alcator-C-Mod. J. Nucl. Mater. 390-391 (1), 339-342. 
TskhakayA, D. 2012 On recent massively parallelized PIC simulations of the SOL. Contrib. Plasma Phys. 52 (5-6), 490-499.

Tskhakaya, D., Subba, F., Bonnin, X., Coster, D. P., Fundamenski, W. \& Pitts, R. A. 2008 On kinetic effects during parallel transport in the SOL. Contrib. Plasma Phys. 48 (1-3), 89-93.

Wootton, A., Carreras, B., Matsumoto, H., Mcguire, K., Peebles, W., Ritz, C., Terry, P. \& ZWEBEn, S. 1990 Fluctuations and anomalous transport in tokamaks. Phys. Fluids B 2 (1990), 2879.

Xu, G., Naulin, V., Fundamenski, W., Hidalgo, C., Alonso, J., Silva, C., Gonçalves, B., Nielsen, A., Juul Rasmussen, J., Krasheninnikov, S. et al. 2009 Blob/hole formation and zonal-flow generation in the edge plasma of the JET tokamak. Nucl. Fusion 49 (9), 092002.

Xu, X., Xiong, Z., Dorr, M., Hittinger, J., Bodi, K., Candy, J., Cohen, B., Cohen, R., Colella, P., Kerbel, G. et al. 2007 Edge gyrokinetic theory and continuum simulations. Nucl. Fusion 47, 809-816.

Zeiler, A., Drake, J. F. \& Rogers, B. 1997 Nonlinear reduced Braginskii equations with ion thermal dynamics in toroidal plasma. Phys. Plasmas 4 (1997), 2134.

Zocco, A., Loureiro, N. F., Dickinson, D., Numata, R. \& Roach, C. M. 2015 Kinetic microtearing modes and reconnecting modes in strongly magnetised slab plasmas. Plasma Phys. Control. Fusion 57 (6), 065008.

Zocco, A. \& ScheKochinin, A. A. 2011 Reduced fluid-kinetic equations for low-frequency dynamics, magnetic reconnection, and electron heating in low-beta plasmas. Phys. Plasmas 18 (10), 102309.

Zweben, S., Boedo, J., Grulke, O., Hidalgo, C., Labombard, B., Maqueda, R., Scarin, P. \& Terry, J. 2007 Edge turbulence measurements in toroidal fusion devices. Plasma Phys. Control. Fusion 49 (7), S1-S23.

Zweben, S., Maqueda, R., Stotler, D., Keesee, A., Boedo, J., Bush, C., Kaye, S., Leblanc, B., Lowrance, J., Mastrocola, V. et al. 2004 High-speed imaging of edge turbulence in NSTX. Nucl. Fusion 44 (1), 134-153. 